\title{
The periodic table of the elements: the search for transactinides and beyond
}

\author{
Maria Letizia Terranova' ${ }^{1} \cdot$ Odilon A. P. Tavares $^{2}$
}

Received: 6 December 2021 / Accepted: 31 January 2022 / Published online: 26 February 2022

(c) The Author(s) 2022

\begin{abstract}
The periodic table of Mendeleev, initially proposed on the basis of 66 elements, and containing 82 elements at the time of Moseley (1887-1915), describes nowadays 118 elements. The huge challenge of this scientific adventure was, and still is, the development of technologies and methods capable of producing elements of atomic number $Z>103$, known as superheavy elements (SHE), or transactinides. This paper presents a survey of experiments and theoretical approaches that led physicists and chemists of today to discover and characterize a number of SHE isotopes. A glance is also given to the feasibility studies performed by scientists aiming to going beyond $Z=118$, building up further neutron-rich nuclides and reaching the ultimate goal of creating long-living new elements at the edge of the Periodic Table.
\end{abstract}

Keywords Transactinides $\cdot$ Superheavy elements $\cdot$ Nuclear reactions $\cdot$ Radioactive decay $\cdot$ Nuclear stability

\begin{tabular}{|c|c|}
\hline \multicolumn{2}{|c|}{ Abbreviations } \\
\hline $\operatorname{CCSD}(\mathrm{T})$ & $\begin{array}{l}\text { Coupled Cluster approach with Single, Dou- } \\
\text { ble, and perturbative Triple excitations }\end{array}$ \\
\hline DF & Dirac-Fock calculation \\
\hline DCB & Dirac-Coulomb-Breit calculation \\
\hline DFT & Discrete Fourier transform method \\
\hline GSI & $\begin{array}{l}\text { Gesellschaft für Schwerionenforschung } \\
\text { (Darmstadt, Germany) }\end{array}$ \\
\hline IP & Ionization Potential \\
\hline IUPAC & $\begin{array}{l}\text { International Union of Pure and Applied } \\
\text { Chemistry }\end{array}$ \\
\hline IUPAP & $\begin{array}{l}\text { International Union of Pure and Applied } \\
\text { Physics }\end{array}$ \\
\hline JINR & $\begin{array}{l}\text { Joint Institute for Nuclear Research (Dubna, } \\
\text { Russia) }\end{array}$ \\
\hline LBNL & $\begin{array}{l}\text { Lawrence Berkeley National Laboratory } \\
\text { (Berkeley, USA) }\end{array}$ \\
\hline
\end{tabular}

This peer-reviewed paper belongs to the Topical Collection "The Periodic System" In celebration of the 150th birth anniversary of sir Ernest Rutherford, the father of Nuclear Physics.

Maria Letizia Terranova

terranova@roma2.infn.it; mleterranova@gmail.com

1 Dipartimento di Scienze e Tecnologie Chimiche, Università Degli Studi di Roma "TorVergata", Via della Ricerca Scientifica s/n, 00133 Rome, Italy

2 Centro Brasileiro de Pesquisas Físicas-CBPF/MCTI, Rua Dr. Xavier Sigaud 150, Rio de Janeiro, RJ 22290-180, Brazil
LLNL Lawrence Livermore National Laboratory (Livermore, USA)

MCDF Multi-configuration Dirac-Fock method

MNT Multi-Nucleon Transfer reaction

ORNL Oak Ridge National Laboratory (Oak Ridge, USA)

QED Quantum electrodynamics

RIKEN Institute of Physical and Chemical Research (Wako City, (Japan)

SHE Superheavy element

\section{What are things made of ?}

The table of the elements released in 1869 by the Russian chemist Dimitri I. Mendeleev (1834-1907) was based on the phenomelogical regularity of the chemical/physical properties exhibited by sixty-six elements known at the time, ordered in function of their atomic weights (Mendeleev 1869). It was only in 1913, from a series of experiments on X-ray diffraction on thirty-nine elements, from aluminum to gold, that the English physicist Henry G. J. Moseley (1887-1915) found the precise physical meaning of atomic number, $Z$, i.e., the number that represents the units of elementary electric charge in the atomic nucleus, therefore the number of electrons in the atom. Consequently, the chemical/physical properties of the elements came to be 
understood as periodic functions of the atomic number, and not of the atomic weight (Moseley 1913).

The ordering scheme proposed by Mendeleev and improved by Moseley gets a scientific foundation during the first decades of the twentieth century, when it was discovered that atomic nuclei consist of protons and neutrons and the quantum mechanical description of the atomic structure was put forward. The advent of the new quantum mechanics in the mid-1920s and the application to electrons moving around the atomic nucleus made possible to describe the periodic table of the elements in terms of atomic structure.

In this context, it became evident that the chemical behavior of an element was driven by the ground-state electronic configuration, i.e., the distribution of electrons density in space. The probability distributions of the electrons in atoms, the so-called "orbitals", indicate the regions of space around the nucleus where the electrons are most likely to be found. In quantum theory applied to the structure of atoms, any electron is identified by a set of four quantum numbers. The principal quantum number, $n=1,2,3,4,5,6$, and 7, indicates the energy level and is associated with the periods of the periodic table of the elements. The orbital quantum number, $l=0$ (orbital s), 1 (orbitals p), 2 (orbitals d), 3 (orbitals f), 4 (orbitals g), $\ldots, n-1$, defines the spatial distribution of the electron. The magnetic quantum number, $m_{l}=l, l-1, \ldots .0, \ldots .,-l+1,-l$, differentiates the behavior of the electron in presence of a magnetic field. Finally, the spin quantum number, $m_{s}=+1 / 2,-1 / 2$, indicates the direction of the intrinsic electron rotation around its axis. The set of these four quantum numbers, with the limitation arising from the "Pauli exclusion principle", enables to completely label an electron in any atom (Quagliano and Vallarino 1973).

However, starting in the 1960s, it was necessary to include also relativistic considerations in the description of the electronic configuration of the heavier elements to explain the chemica/physical properties of new-found natural occuring elements or to predict the behavior of the artificial ones (Pyykkö and Desclaux 1979). Relativistic effects increase with $Z^{2}$, and deviations in the periodicity of the chemical properties were indeed predicted for elements with $Z>100$. For these elements the electrons in the orbitals more close to the highly charged nucleus experience indeed stronger Coulomb forces, and thus are accelerated to relativistic velocities. This primary relativistic effect leads to a stronger electron binding in atoms, causes contraction of the $\mathbf{s}$ and $\mathbf{p}$ orbitals, induces a more efficient screening of the nuclear charge and a corresponding destabilization of the outer $\mathbf{d}$ and $\mathbf{f}$ orbitals. At the same time, spin-orbit interactions occur.

The effects of relativistic electron motion on the spin of electrons modify the orbital quantum number $l$, causing a splitting of the three isoenergetic $\mathbf{p}$ levels and the distribution of electrons into the more stabilized $\mathbf{p}_{1 / 2}$ subshell and the less stabilized $\mathbf{p}_{3 / 2}$ ones (Pyykkö 1988; Eliav et al. 2015; Pershina 2019). The same effects were found to affect also the $\mathbf{d}$ orbitals, inducing a splitting of the isoenergetic shells. As a whole, relativistic effects lead to strong changes in orbital geometry as well as in density and binding energy of electrons in atoms with larger nuclear charge.

Whereas relativistic effects are important in defining the electronic structure and, therefore, the chemical behavior of the superheavy elements, also a feature on nuclear nature must be considered. A property that distinguishes all the elements with $Z>82$ is their instability against radioactive decays, occurring mainly through the processes of alphaparticle emission (Deng et al. 2020) and spontaneous fission (Xu et al. 2008). The emission of nuclear clusters heavier than the alpha-particle can also occur in a number of heavy nuclei (Tavares and Medeiros 2012; Anjali et al. 2020; Santhosh and Nithya 2018).

\section{The survival of the elements}

The stability of a nucleus was for a long period accounted for in the frame of the liquid-drop model (Gamow 1930), that allowed to calculate the binding energy (von Weizsäcker 1935) and to describe the features of the spontaneous fission (Bohr and Wheeler 1939). Following this model, the fissility of a nucleus was expected to increase proportionally to parameter $Z^{2} / A$ ( $A$ denotes the mass number, the sum of the $Z$ protons and $N$ neutrons in a nucleus). With the increased number of protons in a nucleus, the repulsive Coulomb forces overcome the attractive nuclear forces, inducing a nuclear disturbance and finally, just beyond element $Z=100$, the vanishing of the fission barrier height. In this view, the upper limit of the table of the elements was predicted close to element $Z=100$.

However, from 1962 on, several contradictions with the predictions from the liquid-drop model have been observed in fission experiments, and the macroscopic concept of a nucleus as a structureless liquid drop had to be corrected by introducing shell effects. An exciting scenario regarding nuclear stability had been indeed opened by macroscopic-microscopic theories that included also higher orders of nuclear deformations (Strutinsky 1968; Dobaczewski et al. 1984; Myers and Swiatecki 1997).

Following these approaches, the motion of a single nucleon in a largely deformed heavy nucleus undergoing fission is coupled with the collective degrees of freedom of the whole system. The drop-liquid model was thus corrected taking into account effects of independent particles in a medium nuclear field and the existence of closed-shell configurations (Brack et al. 1972). Detailed calculations of the nuclear energy confirmed that shell structures are preserved 
also in highly deformed fissioning nuclei, pointing out that new nuclear shell closures are generated in presence of specific "magic" numbers of protons and neutrons. Under such conditions, an increase of the fission barrier and, therefore, an enhanced stability against nuclear decays were theoretically foreseen (Strutinsky 1968; Brack et al. 1972).

These strong shell effects led to predict the existence of regions in the chart of the nuclides where an enhancement of stability could be expected (Sobiczewski et al. 1966; Myers and Swiatecki 1966; Viola and Seaborg 1966; Strutinsky 1967; Mosel and Greiner 1969; Bolsterli et al. 1972).

The concept of the so-called "island of stability", had been firstly advanced by the North American chemist Glenn T. Seaborg (1912-1999) in 1945 (Seaborg 1945), and further developed by taking into account also nuclear deformations (Sobiczewski 2011; Raeder et al. 2018). The experimentally observed high-fission barrier of the stable "doubly magic" ${ }^{208} \mathrm{~Pb}(Z=82, N=126)$ was rationalized when abandoned the assumption that the large deformation suffered by the nucleus undergoing fission would distruct shell structures.

Theoretical approaches and experimental findings locate the stability islands at values of $Z$ and $N$ different for spherical and for deformed nuclei. In the region of superheavy nuclei, closed shells are expected to occur in spherical nuclei having $Z=114,120$ or 126 and $N=184$, and in deformed nuclei having $Z=108$ and $N=162$ (Türler and Pershina 2013). The topic of nuclear shapes is of fundamental importance in defining the physical/chemical properties of elements, because the distortion from spherical to spheroidal features affects not only the stability against nuclear decays, but also the electronic structure and, therefore, the chemistry of the element (Raeder et al. 2018).

In Fig. 1 are displayed the naturally existing nuclei, as well as the regions where the coupling on $N$ and $Z$ magic numbers predicts an enhanced stability against nuclear decay. Figure 1 indicates also the explosive hydrogen burning on novae or neutron stars surfaces (rp process) and the rapid neutron capture ( $\mathrm{r}$ process) that, along with the s process, are regarded as the fundamental pathways for creation of the elements in the Universe.

These considerations were behind the concept that also elements considered totally instable according to the liquiddrop model of fission, could conversely exist and that the limits of the table of the elements could be overcome (Cwiok et al. 2005; Dean 2007). Even if scientists are still trying to fully understand properties of atoms and nuclei, the theoretical models of nuclear structure developed in the 1960s and 1970s have been a strong boost toward the discover of new heaviest elements.

In accordance with the concept that closed nuclear shells enhance the nuclear stability, the last stable natural element is lead $(\mathrm{Pb})$ formed by 82 protons and 126 neutrons. Going on along the sixth period of the periodic table, the

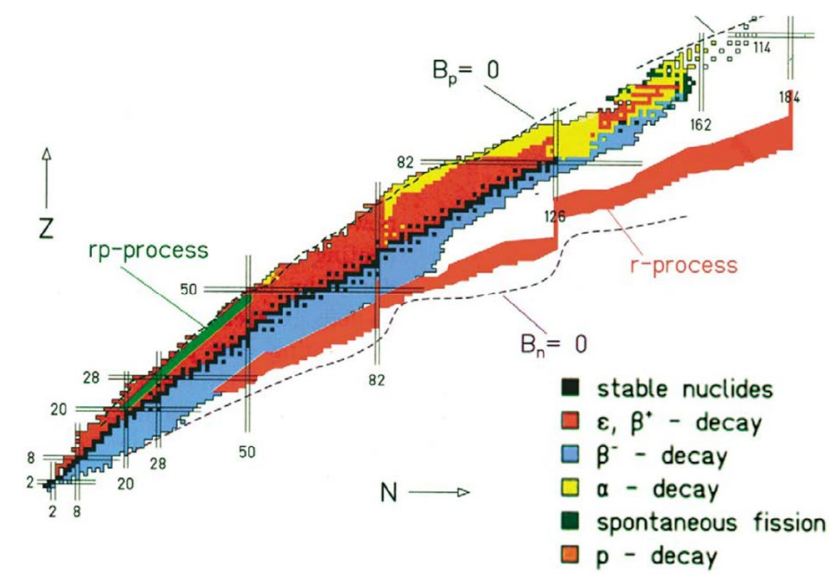

Fig. 1 Map showing the naturally existing nuclei on the Earth (black squares). The magic numbers associated with increased stability (closed shells) are indicated by double lines. The dashed curves show the calculated borderlines of nuclear stability, where the proton and neutron binding energies $B_{\mathrm{p}}$ and $B_{\mathrm{n}}$ become zero. Also shown are the $\mathrm{r}$ process and rp process, two paths believed to be important in the creation of elements of the Universe (Reproduced with permission from Broglia 2002, pag. 775)

proton/neutron ratios do not more enable the physical stability of nuclides, that decay with half-lives, $T_{1 / 2}$, ranging from the quasi-stable ${ }^{209} \mathrm{Bi}\left(Z=83, T_{1 / 2}=1.0 \times 10^{19}\right.$ year $)$ (Tavares et al. 2005) to the relatively short-lived Polonium (Po, $Z=84$ ), Astatine (At, $Z=85$ ), and Radon (Rn, $Z=86$ ) isotopes, for which $T_{1 / 2}$ is in the range $0.27 \mu \mathrm{s}-125$ year (Kondev et al. 2021).

All the elements heavier than $\mathrm{Pb}$ are radioactive and decay by a sequence of $\alpha$ - and $\beta$-particle emissions to the stable $\mathrm{Pb}$. In addition, also the elements of the $4 \mathrm{f}$-block, from $\mathrm{La}(\mathrm{Z}=57)$ to $\mathrm{Lu}(\mathrm{Z}=71)$, known as lanthanides or rare earth elements, have some radioactive isotopes, namely ${ }^{144} \mathrm{Nd}$ (Neodymium, $\left.Z=60\right),{ }^{147,148} \mathrm{Sm}$ (Samarium, $Z=62$ ), ${ }^{151} \mathrm{Eu}$ (Europium, $Z=63$ ), ${ }^{152} \mathrm{Gd}$ (Gadolinium, $\left.Z=64\right)$, and ${ }^{176} \mathrm{Lu}$ (Lutetium, $Z=71$ ) (Kondev et al. 2021).

The seventh period, after the metals $\operatorname{Fr}(Z=87)$, Ra $(Z=88)$ and Ac $(Z=89)$, continues with the elements of actinide series, characterized by the gradual filling of $5 \mathrm{f}$ orbitals. Up to $U(Z=92)$, all the actinides are naturally occurring, whereas the transuranium elements are artificially produced using various nuclear reactions. It should be noted that generally in the tables of elements both Tc $(Z=43)$ and Pm $(Z=61)$ are marked as artificial, because sizeable quantities of these elements have been not found in nature. However, very small amounts of both Tc and Pm are naturally produced through spontaneous fission process in $\mathrm{U}$ isotopes (see, for instance, Rao and Kuroda 1966).

All the actinides are radioactive. The nuclear stability increases from the first members of the actinide family, with $T_{1 / 2}=21.77$ year for ${ }^{227} \mathrm{Ac}, T_{1 / 2}=32.65 \times 10^{3}$ year for ${ }^{231} \mathrm{~Pa}$, up to $T_{1 / 2}=4.46 \times 10^{9}$ year for ${ }^{238} \mathrm{U}$, and $1.40 \times 10^{10}$ year for 
${ }^{232}$ Th (Kondev et al. 2021). The rather long half-lives for these two latter elements, which were produced by nucleosynthesis processes at different times after the Big Bang, explain their current presence on the Earth. Also, traces of the primordial isotopes ${ }^{237} \mathrm{~Np}\left(T_{1 / 2}=2.14 \times 10^{6}\right.$ year $)$ and ${ }^{244} \mathrm{Pu}\left(T_{1 / 2}=8.13 \times 10^{7}\right.$ year $)$ have been detected in nature (Hoffman et al. 1971). In this context, Pu, generally considered an artificial element, can be seen as the heaviest naturally occurring element.

The transuranium elements show a drastic decrease of nuclear stability with increasing the atomic number. The elements with $Z>96$ have relatively short lifetimes, varying from $1.38 \times 10^{3}$ year for ${ }^{247} \mathrm{Bk}$ (Berkelium, $Z=97$ ) down to $22.9 \mu$ s for ${ }^{254} \operatorname{Rf}$ (Rutherfordium, $Z=104$ ) (Kondev et al. 2021). As an example, from ${ }^{238} \mathrm{U}$ isotope to the ${ }^{252}$ No isotope, the addition of 10 protons and 4 neutrons reduces $T_{1 / 2}$ by a factor $10^{16}$. Further studies on rare earth have been reported in (Ascenzi et al. 2020).

\section{Toward new elements}

The era of man-made elements started when the opportunities provided by nuclear processes to modify the properties of the existing nuclei were disclosed. In particular, the long history of transuranium actinides began in 1938 with the discovery of nuclear fission and with the Fermi's guess that such process could produce still unknown nuclides (Pearson 2015). Fission was indeed the first of the standard nuclear reactions used so far to obtain artificial atoms. The other processes were fragmentation of heavy nuclei, limited however to production of elements lighter than $\mathrm{U}$, and fusion reactions, the unique processes able to synthesize superheavy elements (Oganessian and Utyonkov 2015).

Figure 2 displays the regions of the Chart of the Nuclides that can be populated by nuclei produced by fission, fragmentation of fusion processes. These last two processes replicate in laboratory the astrophysical $r$ process paths, that proceed on the neutron-rich side of the $N / Z$ plot (Adamian et al. 2020a). The elements Np, Pu, Am, Cm, Bk, Cf, Es, Fm, $\mathrm{Md}, \mathrm{No}$, and $\mathrm{Lr}$ were obtained by nuclear reactions settled in the period from 1940 (Neptunium, $\mathrm{Np}, Z=93$ ) (McMillan and Abelson 1940) to 1961 (Lawrenciun, $\mathrm{Lr}, Z=103$ ) (Ghiorso et al. 1961), using both neutron or charged particle accelerated beams to bombard targets of lighter actinides (Seaborg 1963).

The chemical/physical characterization performed in 1961 revealed for Lr the ground-state electronic structure $[R n] 5 \mathrm{f}^{14} 7 \mathrm{~s}^{2} 7 \mathrm{p}^{1}$, experimentally confirming the prediction by Seaborg who, in 1949, had indicated element 103 as the final member of the actinides (Silva 2006). In that view, Lr, that completes the 5-f block, even then supported the foresights that the principles governing the periodic trends in

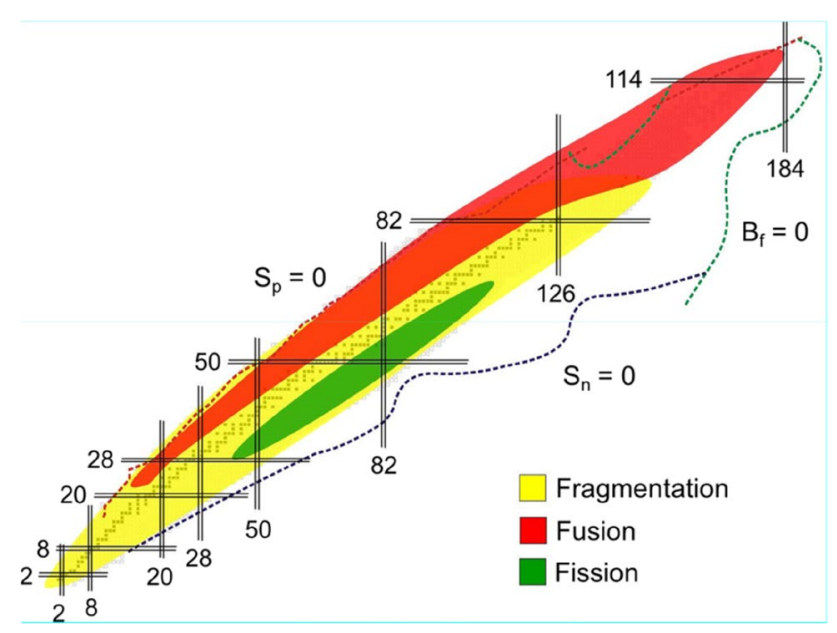

Fig. 2 Chart of the nuclides with indication of the main mechanisms for production of artificial isotopes. Fragmentation reactions produce both neutron-rich and neutron-deficient nuclei up to uranium (yellow area). Fission reactions lead to relatively neutron-rich, intermediate heavy nuclei (green area). Fusion reactions enable to synthesize transuranium and superheavy nuclei, but rather in the neutron-deficient region with respect to the stability line (red area) (Reproduced with permission from Adamian et al. 2020a, page 2) (Color figure online)

groups and periods could be valid for a further stretching of the Periodic Table itself. From then the investigation of still undiscovered elements at the far end of the periodic table began to be a relevant research topic.

The artificial $\mathrm{Lr}$ is not just the last of the actinides, but also the last of the elements described in the majority of the chemistry textbooks. This because only up to $Z=103$ the chemical/physical behavior has been fully investigated and the elements proved to be of practical use. However, generally unoticed by non-experts, and also by chemists, in the last decades, a further outstanding extension of the periodic table has occurred. Based on theoretical predictions and on the hypothesis that the list of chemical elements could be expanded beyond $\mathrm{Lr}$, several nuclear laboratories finalized scientific plans for the synthesis of elements of $Z>103$, the so-called transactinides, or superheavy elements (SHEs).

The developments of particle accelerators have enabled to produce intense beams of stable isotopes for bombarding targets of either stable heavy elements or radioactive actinide isotopes, triggering in such a way a variety of fusion reactions. A long series of nuclear processes induced by accelerated ion beams were carried out at the Joint Institute for Nuclear Research-JINR, Dubna (Russia), at Gesellschaft für Schwerionenforschung-GSI, Darmstadt (Germany), at Lawrence Berkeley National Laboratory-LBNL, Berkeley (USA), at Lawrence Livermore National Laboratory-LLNL, Livermore (USA), and at Institute of Physical and Chemical Research-RIKEN, Wako City (Japan).

A successful series of nuclear physics experiments performed in the 1970s and 1980s allowed to synthesize 
elements from $Z=104$ (Rutherfordium, Rf) up to $Z=108$ (Hassium, Hs). In the following years, the research went ahead, demonstrating that the seventh period of the Table of the Elements could be extended (Münzenberg 1988), and the synthesis of new elements was successfully accomplished up to $Z=118$ (Oganesson, $\mathrm{Og}$ ) (Oganessian and Rykaczewski 2015). These challenging experiments allowed to expand the seventh period of the pariodic table with elements of $104 \leq Z \leq 118$.

All the isotopes of these superheavy elements are instable and characterized by short half-lives, ranging from $10 \mu$ s for ${ }^{267} \mathrm{Ds}$ (Darmstadtium, $Z=110$ ) up to $29 \mathrm{~h}$ for ${ }^{268} \mathrm{Db}$ (Dubnium, $Z=105$ ) (Kondev et al. 2021). For these nuclei, the commonly observed decay modes have been $\alpha$-decay and spontaneous fission. The alpha-decay mode represents the main de-excitation mechanism, competing with spontaneous fission in $20 \%$ of the superheavy isotopes (Magill et al. 2018). In addition, systematic calculations of half-lives for all possible hadronic decay modes, including also the decay by clusters emission (Tavares and Medeiros 2012), have indicated a similar behavior for the isotopic sequences $110 \leq Z \leq 133$ (Duarte et al. 2004), $104 \leq Z \leq 124$ (Poenaru et al. 2012), and $120 \leq Z \leq 126$ (Seyydi 2021).

The success of the experiments aimed at the discovery of SHEs strongly relied on the ability to identify their shortlived isotopes. A first difficulty arises from the very low cross sections of the synthesis processes, from $\mathrm{nb}\left(10^{-33}\right.$ $\left.\mathrm{cm}^{2}\right)$ to $\mathrm{pb}\left(10^{-36} \mathrm{~cm}^{2}\right)$ or even $\mathrm{fb}\left(10^{-39} \mathrm{~cm}^{2}\right)$, that enable the production of no more than one atom-at-time. Moreover, the identification of these rare events is made harder because the fusion reactions that produce the transactinides are accompanied by a variety of nuclear processes, resulting in a simultaneous abundant production of elements all over the periodic table (Itkis et al. 2002). A decisive role in overcoming such technical challenges has been played by the avaibility of sophysticated physical and chemical methodologies and of highly sensitive nuclear instrumentation.

To separate the highly charged nucleus of SHE generated by a complete fusion reaction from the by-products, a pre-separation step of the reaction products that recoil out from the target is accomplished using various highly performing electrostatic and magnetic separators. To further eliminate the interferences of other elements and to allow an unambiguous detection of the searched atoms, chemical separations using thermochromatography and isothermal gas chromatography, along with ion exchange chromatography and extraction chromatography, have been, and still are, the most employed methodologies (Türler et al. 2015; Nagame et al. 2015). A detailed description of the instrumentation utilized for single atom chemistry can be found in Türler and Pershina (2013).

The presence of the complete fusion product is then proved by the detection of the characteristic nuclear signals generated by the atom itself and by the chain of its sequential radioactive decays. This step, accomplished in a timecorrelated event-by-event mode, makes use of high-resolution spectrometers for the detection of correlated emission of alpha particles and fission fragments. In this way, the elements with $Z$ from 104 to 118 are now well established inside the periodic scheme proposed by Mendeleev, even if the relativistic effects introduced some irregularities in the trends of chemical and physical properties (Pyykkö 2011; Eliav et al. 1998b). The deviations in the periodicity of chemical properties, deeply illustrated in Eichler (2019b) and Pershina (2020), regard mainly bond character, ionization potentials, oxidation states, and enthalpies of compound formation.

Taking into account, the intrinsic difficulties of this kind of research, could be expected either discussions about experimental findings and related interpretations or arguments about the priority of the discovery by different competing scientific teams. The emergence of controversies occurred just from the beginning, and involved several of the elements artificially produced in the 20th and in the present centuries (Kragh 2018). This is the reason why the International Union of Pure and Applied Chemistry-IUPAC and the International Union of Pure and Applied Physics-IUPAP in some cases delayed to acknowledge to a team the priority of a new element discovery.

So far, the elements from 104 to 118 have been all authenticated by IUPAC and IUPAP, who made official for the transactinides the following names, proposed by the credited discoverers (see Appendix 1): Rutherfordium (Rf, $Z=104$ ), Dubnium (Db, $Z=105$ ), Seaborgium (Sg, $Z=106$ ), Bohrium (Bh, $Z=107$ ), Hassium (Hs, $Z=108$ ), Meitnerium (Mt, $Z=109$ ), Darmstadtium (Ds, $Z=110$ ), Roentgenium ( $\operatorname{Rg}, Z=111)$, Copernicium (Cn, $Z=112$ ), Nihonium (Nh, $Z=113$ ), Flerovium (Fl, $Z=114$ ), Moscovium (Mc, $Z=115)$, Livermorium ( $\mathrm{Lv}, Z=116)$, Tennessine (Ts, $Z=117)$, and Oganesson (Og, $Z=118)$. Presently, 118 elements and 3340 nuclides in the ground state (of which 254 are stable isotopes) have been authenticated (Kondev et al. 2021).

\section{Elements 104-118: the discovering}

Fusion reactions occurring when thin targets of heavy elements are bombarded with highly energetic ion beams are the only nuclear processes suitable for the production of elements in the transuranium region. The conception behind fusion is to select target and projectile elements so that the sum of their protons could correspond to the $Z$ of the planned new element. To carry on a fusion process, the coulomb repulsion ion/nucleus has to be overcome and this requires projectile energies of about $5 \mathrm{MeV} /$ nucleon. In this 
view, large accelerators are needed to accomplish the transactinides, that in any case are obtained with cross sections values not exceeding the pb or, for the heavier elements, even the $\mathrm{fb}$ range (Adamian et al. 2020b). The production of elements 104-106 had been achieved by irradiating thin targets made by isotopes of the transuranium elements $\mathrm{Pu}, \mathrm{Cf}$, $\mathrm{Bk}$, and Am with high-energy beams of light ions, mainly $\mathrm{C}, \mathrm{N}, \mathrm{O}$, and $\mathrm{F}$.

Element 104 (Rf) was discovered in 1964 at JINR when the irradiation of a ${ }^{242} \mathrm{Pu}$ target by $115-\mathrm{MeV}^{22} \mathrm{Ne}$ ions produced the isotope ${ }^{260} \mathrm{Rf}\left(T_{1 / 2}=0.3 \mathrm{~s}\right)$ (Flerov et al. 1964), whereas the element $105(\mathrm{Db})$ was identified in 1971 at LBNL by reactions of highly energetic ${ }^{18} \mathrm{O}$ and ${ }^{16} \mathrm{O}$ beams with ${ }^{249} \mathrm{Bk}$ targets (Ghiorso et al. 1971).

The element $106(\mathrm{Sg})$ was independently synthesized in 1974 at LBNL by the group leadered by Seaborg, who produced the element by the fusion of ${ }^{18} \mathrm{O}$ and ${ }^{249} \mathrm{Cf}$ (Ghiorso et al. 1974), and at JINR by Oganessian and coworkers by the reaction of low-energy ${ }^{54} \mathrm{Cr}$ beams with ${ }^{207} \mathrm{~Pb}$ targets (Oganessian et al. 1974). This last experiment was very important, because evidenced how new target/projectile combinations, different from those until then considered, were needed to enhance the production yield of the more heavy transactinides. As a matter of fact, the new approach demonstated the ability to increase the survival probability of the fusion products.

The fusion of accelerated light ions and heavy elements produces indeed a superheavy, highly excited compound nucleus with energy in the range $40-50 \mathrm{MeV}$. Such hot nucleus promptly cools down by evaporation of a variable number of neutrons, thus generating numerous different isotopes, the so-called fusion-evaporation residues. Moreover, the cooling step by nucleon evaporation is a process that competes with spontaneous fission, more probable in more excited nuclei. As a result of such fusion-fission reactions induced by light-ion beams, the forecasts were that the survival of the superheavy element would be strongly reduced and that any elements with $Z>106$ would be hardly obtained in measurable amounts. Scientists at JINR demonstrated that using for the fusion targets from the $\mathrm{Pb}$ region and beams of neutron-rich isotopes of $\mathrm{Ti}$ (Titanium), $\mathrm{Cr}$ (Chromium), and $\mathrm{Fe}$ (Iron), it was possible to create less excited compound systems (Oganessian et al. 1976).

Even if the cross sections for fusion based on heavier projectiles were significantly smaller, the low excitation energy of the compound nuclei was found to allow the evaporation of only one or two neutrons and, consequently, to reduce the probability of decay by spontaneous fission. This effect has proved to be even more important in the progressive advance along the transactinide row, and the new "cold-fusion" approach was widely exploited in the successive experiments that led to the discovery of 107-109 elements. The first isotope of element $107(\mathrm{Bh})$, the short-lived metastable ${ }^{262} \mathrm{Bh}^{\mathrm{m}}$, was identified at GSI in a fusion reaction of low-energy ${ }^{54} \mathrm{Cr}$ ions and ${ }^{209} \mathrm{Bi}$ targets (Münzenberg et al. 1981).

The reaction of ${ }^{58} \mathrm{Fe}$ beams with a ${ }^{208} \mathrm{~Pb}$ target led in 1984 to the discovery of element 108 (Hs) (Münzenberg et al. 1984). The evaporation of one neutron from the compound nucleus with $18-\mathrm{MeV}$ energy produced the ${ }^{265} \mathrm{Hs}$ isotope ( $T_{1 / 2}=1.5 \mathrm{~ms}$ ). The ${ }^{269} \mathrm{Hs}$ isotope with $T_{1 / 2} \approx 14 \mathrm{~s}$, more suitable for chemical investigations, was later produced by the $\alpha$-particle decay chain originating from the reaction of ${ }^{208} \mathrm{~Pb}$ with ${ }^{70} \mathrm{Zn}$ ions beams (Hofmann et al. 2002). The research team at GSI had identified in 1982 element 109 (Mt) by $\alpha$-decay of a single atom of the ${ }^{267} \mathrm{Mt}$ isotope produced by the fusion of ${ }^{58} \mathrm{Fe}$ ions with ${ }^{209} \mathrm{Bi}$ (Münzenberg et al. 1982).

Following the same methodology, in the 1990s, a series of experiments allowed to discover elements from 110 to 112 . Ds $(Z=110)$ was created in 1994 by bombardment of ${ }^{208} \mathrm{~Pb}$ with accelerated ${ }^{62} \mathrm{Ni}$ ions. The process produced a single atom of the isotope ${ }^{269} \mathrm{Ds}$, identified by measuring 4 genetically linked alpha-decay chains (Hofmann et al. 1995a). The production cross section of the Ds isotopes has been after that increased from $3.5 \mathrm{pb}$ up to $15 \mathrm{pb}$ by the use of ${ }^{64} \mathrm{Ni}$ ions.

The first isotope of element $111(\mathrm{Rg})$ was identified from the detection of 3 alpha-decay pattern. For the isotope ${ }^{272} \mathrm{Rg}$, produced via the ${ }^{64} \mathrm{Ni}+{ }^{209} \mathrm{Bi} \rightarrow{ }^{272} \mathrm{Rg}$ fusion reaction, a cross section of $3.5 \mathrm{pb}$ was deduced (Hofmann et al. 1995b). First results for the synthesis of element 112 (Cn) were reported in 1996 (Hofmann et al. 1996). Even with the most intense beam the reaction of ${ }^{70} \mathrm{Zn}$ with ${ }^{208} \mathrm{~Pb}$ yielded only one atom of the ${ }^{277} \mathrm{Cn}$ isotope in 1 week. In 2000, a second experiment at GSI allowed to detect a second atom (Hofmann et al. 2002). Two more ${ }^{277} \mathrm{Cn}$ atoms were produced in 2004 at RIKEN (Morita et al. 2007). Taken together, this set of results allowed to confirm the creation of element Cn (Hofmann 2010).

The search for element $113(\mathrm{Nh})$ started in 2003 at RIKEN using again a ${ }^{70} \mathrm{Zn}$ ion beam and a ${ }^{209} \mathrm{Bi}$ target. About 580 days of beam irradiations performed from 2003 to 2012 allowed to produce few atoms of the 113 element, identified through three decay chains of its 278 isotope (Morita 2015). The element was recognized by IUPAC in 2016 (Karol et al. 2016), and named Nihonium from "Nihon", a translation of Chinese characters meaning "the land of rising sun", the historical name for Japan (En'yo 2019). The extremely low value ( $20 \mathrm{fb})$ of cross section for ${ }^{278} \mathrm{Nh}$ production was a clear signal that the cold-fusion approach was no more valid to generate elements of $Z>113$ (Hofmann 2016).

The synthesis of the elements from 114 up to 118 , as well as of various isotopes of the elements 112 and 113, was made possible by a new synthesis methodology settled at JINR in the 1990s (Oganessian and Utyonkov 2015). This strategy envisaged the irradiation of targets made by 
radioactive actinides, from ${ }^{238} \mathrm{U}$ up to ${ }^{249} \mathrm{Cf}$, by highly accelerated ${ }^{48} \mathrm{Ca}$ ions.

The design of the new experiments was based on the need to produce neutron-rich fusion products, predicted to have a longer life. In this context the choice has fallen on ${ }^{48} \mathrm{Ca}$, a rather light nucleus characterized by a neutron excess (neutron to proton ratio of 1.4). Therefore, it was expected that the superheavy nuclei created by the fusion of ${ }^{48} \mathrm{Ca}$ isotope with actinide nuclei would be rather close to the neutron-rich region of nuclear stability. The experiments confirmed that the mechanism of such "hot-fusion" processes induced a cooling of the excited compound nucleus through the evaporation of a number of neutrons lower than those emitted in a cold-fusion obtained by low-energy high- $Z$ beams. As a result, the cross sections for the production of transactinide elements could be up to 100 times larger.

However, this hot-fusion methodology required noticeable technical efforts, due to the need to employ projectiles and targets completely different from those utilized for the cold-fusion processes. As regards the ion source, complex enrichment procedures were settled to obtain grams of the ${ }^{48} \mathrm{Ca}$ isotope, present in natural Ca only as $\sim 0.19 \%$. Moreover, remarkable improvements of the irradiation set-up were requested when using targets made by rare isotopes of ${ }^{244} \mathrm{Pu}$, ${ }^{243} \mathrm{Am},{ }^{248} \mathrm{Cm},{ }^{249} \mathrm{Bk}$, and ${ }^{250} \mathrm{Cf}$. Being the actinide isotopes obtained in small amounts by nuclear reactions induced by intense neutron fluxes, care had to be taken to avoid rapid consumption of such valuable targets during long-lasting continuous bombardment by intense $\left(10^{13}\right.$ ions $\left./ \mathrm{s}\right){ }^{48} \mathrm{Ca}$ beams.

Following the hot-fusion approach, element 114 (Flerovium, Fl) has been created by reaction of ${ }^{48} \mathrm{Ca}$ with ${ }^{242} \mathrm{Pu}$ and ${ }^{244} \mathrm{Pu}$ targets, obtaining the isotopes ${ }^{287} \mathrm{Fl}$ and ${ }^{288} \mathrm{Fl}$ that could be identified through 3 and 2 alpha-decay chains, respectively (Oganessian et al. 1999).

The first identification of element 115 (Moscovium, Mc) was made by a joint Dubna-Livermore team in 2003. The research group experienced difficulties in the identification of the four atoms resulting from the fusion of ${ }^{243} \mathrm{Am}$ with ${ }^{48} \mathrm{Ca}$. This was due to the fact that none of the nuclides (Nh, $\mathrm{Db}$ ) originating from the alpha-decay of the produced atoms was known at that time (Oganessian et al. 2004).

Starting from 1977, a lot of unsuccessful attempts characterized the discovery of element 116 (Livermorium, Lv) (Armbruster et al. 1985). It was only in 2000, measuring the decay data of a single atom created by bombarding ${ }^{249} \mathrm{Cm}$ with ${ }^{48} \mathrm{Ca}$, that the presence of the ${ }^{292} \mathrm{Lv}$ isotope was confirmed (Oganessian et al. 2000). In 2012 to element 116 was assigned the name Livermorium (Lv), recognizing the role played by the Lawrence Livermore National Laboratory (LLNL), who had worked with JINR on the discovery of elements 113-116 and 118.
A series of obstacles, in this case mostly of organizational type, were found also in carrying on the experiments for the synthesis of element 117 (Tennessine,Ts), a research carried out in the frame of an JINR-LLNL-ORNL (Oak Ridge National Laboratory) collaboration. To obtain an element with $Z=117$ using a ${ }^{48} \mathrm{Ca}$ beam, the right target must be made of ${ }^{249} \mathrm{Bk}$, an actinide obtained as sub-product of the nuclear process yielding ${ }^{252} \mathrm{Cf}$. However, at that time, ORNL, the only producer of ${ }^{249} \mathrm{Bk}$, could not provide such isotope. So, the experiment launched in 2004 had to be stopped up to the end of 2008, when ORNL made available $22 \mathrm{mg}$ of ${ }^{249} \mathrm{Bk}$. In addition, bureaucratic troubles delayed the shipment of such radioactive material to Dubna, but, finally, the fusion experiments could be performed.

A first identification of element 117 was achieved on the basis of two alpha-decay chains that produced the short-living ${ }^{294} \mathrm{Ts}$ and ${ }^{293} \mathrm{Ts}$ isotopes of element Tennessine (Oganessian et al. 2010). However, the discovery of element 117 was confirmed only later, when all the daughter nuclides produced by the decay chains had been identified (Oganessian et al. 2013; Forsberg et al. 2016). The element was named Tennessine to acknowledge the contribution of the University of Tennessee (USA) in its identification (Khuyagbaatar et al. 2019).

On the other side, the discontinuity in researches for the synthesis of element 117 allowed to put more efforts in experiments aimed to confirm the creation of element 118 . As a matter of fact, in 2002 , the bombardment of a ${ }^{249} \mathrm{Cf}$ target with ${ }^{48} \mathrm{Ca}$ at $245 \mathrm{MeV}$ had produced only one atom. The alpha-decay chain of this atom led to provisionally ascribe it to the element 118 , but the discovery was announced only in 2005, when the use a thicker target and of a higher energy beam allowed to produce two more isotopes of element 118 with mass number 294 (Oganessian 2006). Moreover, when at Dubna in 2012 were going on further irradiations of the finally available ${ }^{249} \mathrm{Bk}$ to produce element 117 , the analysis of alpha-decay chains evidenced also the creation of atoms of element 118 . This unexpected result was due to the $\beta$-decay of ${ }^{249} \mathrm{Bk}$ into ${ }^{249} \mathrm{Cf}$, and prompt production of element 118 instead of the scheduled 117 one (Oganessian and Rykaczewski 2015).

The discovery of element 118 has been recognized by IUPAC and IUPAP in 2016 (Karol et al. 2016). The name Oganesson was given to acknowledge the outstanding researches carried out by the group leadered by the Russian nuclear physicist Yuri Ts. Oganessian (b. 1933), who strongly contributed to the creation of new SHEs by designing advanced set-ups and innovative experiments. The addition of Oganesson, that is presently the last element formally recognized, completed the seventh period of the PeriodicTable.

An overview of the up to date situation regarding SHEs is provided by the portion of the Chart of Nuclides of Fig. 3, 
Fig. 3 Chart of nuclides with the known isotopes of superheavy elements. The contour maps of the blue background outline shell correction energies calculated in the macroscopicmicroscopic model (Möller et al. 2009) The blue darkening indicates increase of fission barrier. The chart shows the island of enhanced stability around $Z=114, N=184$, where spherical shell closures are expected. The violet squares mark still unknown isotopes: some of them could be produced in fusion reactions of ${ }^{248} \mathrm{Cm}$ targets with stable isotopes of the projectile nuclei indicated on the right side (Reproduced with permission from Adamian et al. 2020a, page 39) (Color figure online)

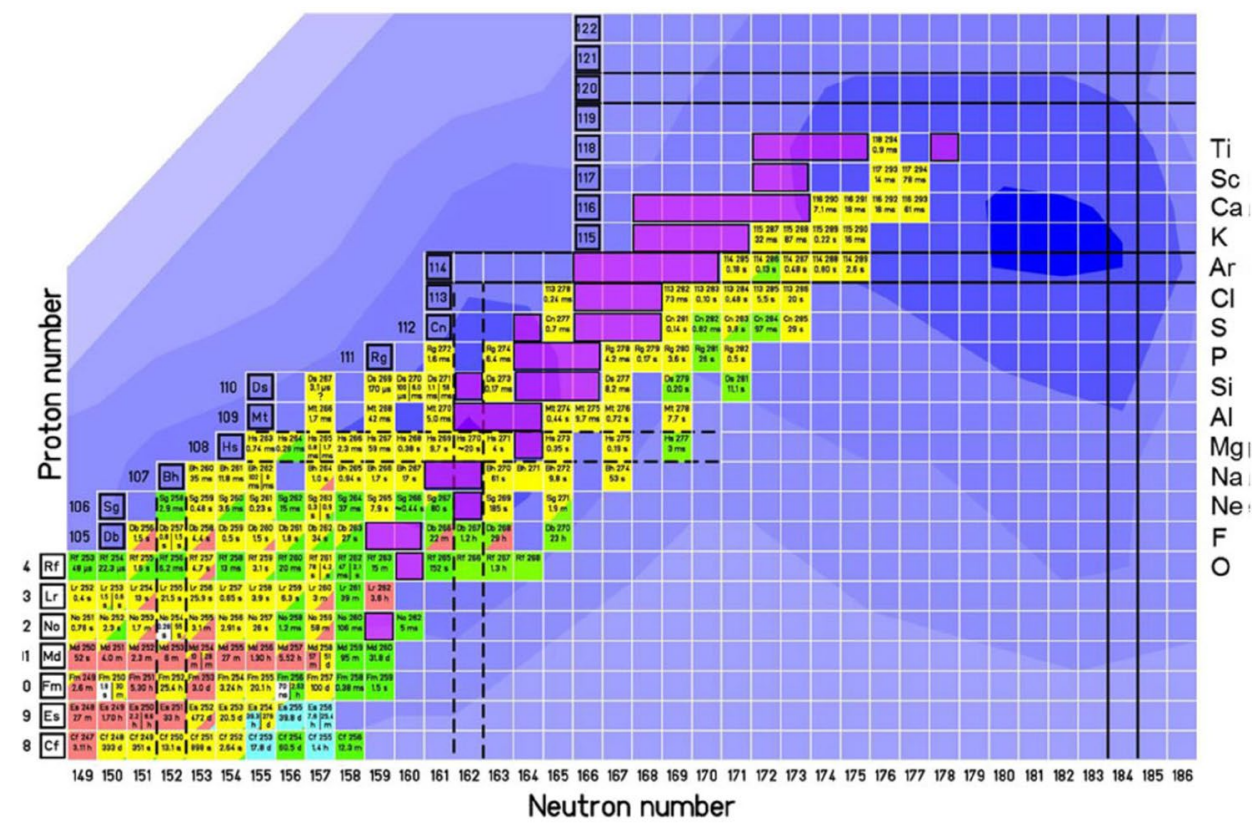

where all the produced isotopes are shown along with indication of their predominant decay modes and half-lives (see also a summary table in Appendix 1).

\section{A look at the chemistry of transactinides}

Given that transactinide atoms are obtained in amounts ranging from 1 atom per day to 1 atom per week, and that the half-lives of a hundred of their isotopes are of the order of seconds (or even microseconds), the investigation of their chemical properties is a very hard, and for some of them really impossible, task. In effect, the chemical experiments had to be performed on "one-atom-at-a-time" and only on isotopes with life-times enabling separation processes in gas or in aqueous phase. Moreover, the investigations are limited to the study of few chemical properties, such as volatility, surface adsorption, redox potentials and ability of complex formation (Gäggler and Türler 2014). As regards this last topic, so far only for few SHEs the chemical complexes, mainly oxyhalides, oxycarboxylates and carbonyl complexes could be studied (Türler and Gregorich 2014; Kratz and Nagame 2014). Details regarding the formation of complexes for the transactinides in various oxidation states are reported in Türler and Pershina (2013).

The present technologies do not make possible experimental assessment of the many other properties strictly associated with the chemical and physical behavior of the SHEs, such as chemical reactivity, excitation energies, ionization potentials and electron affinities. In this context theoretical studies, as ab-initio Dirac-Fock (DF) quasi-relativistic approaches, were found to play a fundamental role. As delineated in the paper by Pershina (2015), molecular calculations not only enabled to insert in the periodic table the elements created up till now, but also to predict a reasonable placement for elements up to $Z=122$.

The coupling of theoretical and experimental approaches is even more significant in interpreting and corroborating the scarce experimental data available on the atomic properties of the SHEs. Of fundamental importance are the calculated ionization potential (IP) for the first and multiple possible ionizations, because such data are used in turn to predict several fundamental chemical and physical properties of the elements and of their compounds (Ginges and Dzuba 2015).

Very accurate calculations have enabled to detail energy and hyperfine structure of atomic states for heavy elements with relatively simple electron structure (Schwerdtfeger et al. 2015; Eliav et al. 2015). Little theoretical data are conversely available on atomic properties of the elements heavier than 105 , for which the complicated electron structure with open $6 \mathrm{~d}$ shell makes it difficult to perform accurate calculations, and approximation methods must be applied (Dzuba 2016).

As regards the positioning of the transactinide elements in the Periodic Table, it has been based on computational analysis and a limited number of experimental observations. However, the obtained results were sufficient to reveal similarities with the chemical behavior of lighter elements and to confirm that the general trends of the periodic properties continued to be still valid, although with some deviations, in the region of superheavy nuclei.

In effect, even if for these elements the relativistic effects induced by the increasingly high nuclear charge strongly influence the electronic configuration (Pershina 2019), the alteration of the electron distribution functions does not 
totally disrupt the established trends of recurrent features along periods and groups. These considerations, along with computational studies, allowed scientists to integrate the scarce experimentally assessable chemical properties of the new elements and to regard them, on a case-by-case basis, as homologous of lighter elements in corresponding groups.

Discrete Fourier transform (DFT) and multi-configuration Dirac-Fock (MCDF) methods demonstrated that the first nine transactinide elements, from $\mathrm{Rf}$ to $\mathrm{Cn}$, have an electronic structure characterized by the progressive filling of $6 \mathrm{~d}$ shell. For $\operatorname{Rf}(Z=104)$, data of orbital energies suggested the ground-state electronic configuration $[R n] 5 f^{14} 7 \mathrm{~s}^{2} 6 \mathrm{~d}^{2}$. The occurrence of the first ionization at expenses of the $6 \mathrm{~d}$ shell indicated that $\mathrm{Rf}$ can be regarded as a transition metal of $6 \mathrm{~d}$ block (Glebov et al. 1989). Similarly to the lighter homologues of the 4-group, Rf was expected to have a chemical behavior characterized by four valence electrons and thus by a stable $4+$ oxidation state. Confirmation has been obtained by comparing the calculated first four ionization potentials of $\mathrm{Rf}$ and of the other 4-group elements, $\mathrm{Hf}, \mathrm{Zr}$ and Ti (Johnson et al. 1990).

An external configuration $6 \mathrm{~d}^{3} 7 \mathrm{~s}^{2}$ has been predicted for the next element, $\mathrm{Db}(Z=105)$, with $5+$ as maximum oxidation state like the homologous of the 5-group. However, relativistic effects are already being felt, as resulted by comparison of the calculated IP values for removal of a $6 \mathrm{~d}$ electron from $\mathrm{Db}$, and of a $6 \mathrm{~s}$ electron from $\mathrm{Ta}$ (Fricke et al. 1993). For $\mathrm{Sg}(Z=106)$, MCDF calculations, confirmed by chemical experiments, suggested the ground-state external configuration $6 \mathrm{~d}^{4} 7 \mathrm{~s}^{2}$ and the belonging to the 6-group, with $\mathrm{IP}=7.85 \mathrm{eV}$ for the loosing of a $6 \mathrm{~d}$ electron and the possibility of multiple ionizations up to a $6+$ state (Johnson et al. 1999).

The element $107(\mathrm{Bh})$, with the calculated ground-state $6 d^{5} 7 s^{2}$, is predicted to be a homolog of Re, Tc and $\mathrm{Mn}$ in the 7-group. From MCDF computing (Johnson et al. 2002) the energy for the first ionization potential of a $6 \mathrm{~d}$ electron is $7.7 \mathrm{eV}$, a value slightly lower with respect to the $7.8 \mathrm{eV}$ for the loosing of a $6 \mathrm{~s}$ electron in Re, confirming the increasing impact of relativistic destabilization and spin-orbit splitting on the d orbitals of the heavier elements. The comparison of predicted IP values for multiple ionizations of transactinides from 3- up to 7-groups, with IP values measured for the respective lighter homologs, evidenced that SHEs exhibit an increased stability of the maximum oxidation states and, conversely, a stability decreasing of the lower states.

At $Z=108$ starts the inversion of the $7 \mathrm{~s}$ and $6 \mathrm{~d}$ energy levels. The ground state of $\mathrm{Hs}$ is $6 \mathrm{~d}^{6} 7 \mathrm{~s}^{2}$. MCDF calculations indicated a value of $7.6 \mathrm{eV}$ for the first ionized electron $6 \mathrm{~d}$, and a multiplicity of oxidation states, up to $8+$ (Johnson et al. 2002).

For some transactinides, the belonging on a group in the Periodic Table has been agreed only on computational basis.
This is the case of Mt, placed in the 9-group even if was not possible to experimentally study its chemistry and to verify similarities with the behavior of the lighter homologs Ir, Rh, and Co. Also, for element 110, Ds, experimental studies are hampered by the very short half-lives of all the produced isotopes. However, significant theoretical investigations allowed to inset elements 109 and 110 in groups 9 and 10, respectively. For Mt was calculated a $6 \mathrm{~d}^{7} 7 \mathrm{~s}^{2}$ configuration, similar to that of Ir. Conversely, it was found that, due to the relativistic effects, the ground state of Ds is $6 \mathrm{~d}^{8} 7 \mathrm{~s}^{2}$, different from that of its lighter homolog $\mathrm{Pt}\left(5 \mathrm{~d}^{9} 6 \mathrm{~s}^{1}\right)$. Also, the configuration $6 \mathrm{~d}^{7} 7 \mathrm{~s}^{2}$ for the first ionizated state of Ds is predicted different from the $5 \mathrm{~d}^{9}$ of $\mathrm{Pt}+$.

The relativistic contraction and stabilization of $7 \mathrm{~s}$ orbital, a phenomenon that reaches a maximum at $Z=112$, is already very effective for element $111(\mathrm{Rg})$. Several computational methods suggested for Rg a ground-state $6 \mathrm{~d}^{9} 7 \mathrm{~s}^{2}$ and a chemistry different from the chemistry of the lighter homolog $\mathrm{Au}\left(5 \mathrm{~d}^{10} 6 \mathrm{~s}^{1}\right)$ of the 11-group. The strong relativistic effects provoke indeed a decrease of $\mathrm{Rg}$ atomic radius and of bond lengths in $\mathrm{Rg}$ compounds, contrasting the trends of an increase of such values with increasing $Z$, established for the homologs of the 11-group. These enhanced effects act also on ionizated states, leading to a configuration of $\mathrm{Rg}+$ $\left(6 d^{8} 7 s^{2}\right)$ different from the $5 d^{10}$ of $\mathrm{Au}+$, and to the stabilization of the higher oxidation states (up to +6 ) in $\mathrm{Rg}$.

At $Z=112$, the filling of the $6 \mathrm{~d}$ orbitals is completed and $\mathrm{Cn}$ is the last of the transition metals belonging to the $6 \mathrm{~d}$ block (Gyanchandani and Sikka 2011). The $6 \mathrm{~d}^{10} 7 \mathrm{~s}^{2}$ closedshell ground state and the maximum of the relativistic stabilization of the 7s orbital led,since 1975, to predict for a 112 element chemical/physical properties very different from those of the homologs in the 12-group (Pitzer 1975). The relativistic coupled cluster method yielded $11.97 \mathrm{eV}$ for the first, and $22.49 \mathrm{eV}$ for the second ionization of $\mathrm{Cn}$. These IP values are the highest not only in the 12-group but in the whole 7th period (Eliav et al. 1995). These data are consistent with an oxidation state zero for $\mathrm{Cn}$. However, the destabilization of the $6 \mathrm{~d}$ electrons and the inversion of $7 \mathrm{~s}$ and $6 \mathrm{~d}$ energy levels enable to foreseen for $\mathrm{Cn}$ also a $2+$ oxidation state at the expense of $6 \mathrm{~d}$ electrons, and an increase of stability for a $4+$ state. $\mathrm{Cn}$ is expected to have an atomic radius of $1.71 \AA$, the smallest in the 12-group, and the shortest bond lengths in compounds. On the whole, the strong relativistic effects induce in $\mathrm{Cn}$ a high chemical inertness, much more pronounced with respect to the lighter homologs in the group (Pershina 2015).

At $Z=113$ starts the progressive filling of the $7 \mathrm{p}$ orbitals, strongly influenced by relativistic effects that increase along the series, whereas the $7 \mathrm{~s}^{2}$ electrons are made rather inactive due to the even large stabilization in elements from 113 to 118. So, even if these elements are placed in the main groups of the Periodic Table where the chemistry is traditionally 
determined by the behavior of "s" and " $p$ " external electrons, for elements from $\mathrm{Nh}$ to $\mathrm{Og}$ the trends of properties already assessed for the lighter homologs are expected to show exciting deviations.

The gas chromatography studies of the $\mathrm{Nh}(Z=113)$ isotopes obtained at RIKEN allowed to suggest the $6 \mathrm{~d}^{10} 7 \mathrm{~s}^{2} 7 \mathrm{p}_{1 / 2}^{1}$ configuration and, therefore, the belonging of $\mathrm{Nh}$ to 13-group, but prevented an unambiguous chemical speciation of the element (Türler et al. 2015). More information on the chemistry of $\mathrm{Nh}$ was obtained later by the Dubna team, that performed adsorption experiments after a physical preparation of Nh samples (Aksenov et al. 2017), finding agreement with the $\mathrm{Nh}$ gas-phase behavior predicted by relativistic density functional theory. However, as recently pointed out by Eichler (2019a), a definitive chemical characterization of $\mathrm{Nh}$ has not yet been reached.

The stabilization of the $7 p$ orbitals and the quasi-closed $7 \mathrm{~s}^{2} 7 \mathrm{p}^{2}{ }_{1 / 2}$ ground-state structure induced by the spin-orbit coupling suggested that element $114(\mathrm{Fl})$ could have a behavior similar to that of an inert gas, and a zero electron affinity (Borschevsky et al. 2009). Recent calculations give for the first ionization potential a value of $8.626 \mathrm{eV}$, evidencing an inversion in the trend of IP values of 14-group. Such values, after the decrease from $\mathrm{C}$ to $\mathrm{Sn}$, start to increase again from $\mathrm{Sn}$ to Fl. Experiments of $\mathrm{Fl}$ adsorption on $\mathrm{Au}$ confirmed the general trend of decreasing metallic character going down along 14-group, and the reaching for $\mathrm{Fl}$ of a maximum oxidation state of $2+$. Interesting, the data achieved by challenging chemical experiments indicated a totally new state for Fl, featuring properties of a gaseous noble metal.

In the case of $\mathrm{Mc}(Z=115)$, the limited production of its short-lived isotopes has hampered so far experimental measurements, and the properties of the element and its compounds can be only theoretically predicted (Pershina 2014). The proposed ground-state configuration $7 \mathrm{~s}^{2} 7 \mathrm{p}^{2}{ }_{1 / 2} 7 \mathrm{p}^{1 / 2}$ indicates that Mc can be considered an element of 15-group. However, notable differences with the chemistry of lighter homologs $\mathrm{Bi}$ and $\mathrm{Sb}$ are likely to arise from the lowering of the $7 \mathrm{~s}$ and the $7 \mathrm{p}$ orbitals' energy and, moreover, by the sub-shell splitting of these last electronic levels (Faegri and Trond 2001; Santiago and Haiduke 2020). In effect, Mc is characterized by two inert electron pairs $\left(7 \mathrm{~s}^{2}\right.$ and $\left.7 \mathrm{p}^{2}{ }_{1 / 2}\right)$ and by one $7 \mathrm{p}^{1}{ }_{3 / 2}$ electron at the higher energy. This would explain the rather low IP value of $5.579 \mathrm{eV}$ obtained from DCB calculations (Eliav et al. 1998b). Overall, Mc can be considered to have a predominant metallic character, with $\mathrm{Mc}+1$ as the more stable oxidation state, and $\mathrm{Mc}+3$ as the only other possible state.

Experimental difficulties have been faced also in the investigation of elements 116 and 117, for which there is still a lack of experimental data. For $\mathrm{Lv}(Z=116)$, recent calculations performed in the framework of the Dirac-Coulomb
Hamiltonian, and corrected for the Breit and quantum electrodynamics (QED) contributions. provided accurate values of IP for the elements and for its ions (Borschevsky et al. 2015). The computed data of energies and IPs values of Lv and $\mathrm{Lv}^{+}$evidenced a strong influence of the electron correlation effects.

As regards element 117 (Ts) with the external electron configuration $7 \mathrm{~s}^{2} 7 \mathrm{p}_{1 / 2}^{2} 7 \mathrm{p}_{3 / 2}^{3}$, a behavior significantly different from those of the homologous of the 17-group has been predicted. However, the lack of experimental data did not enable so far to define any chemical/physical property. Based on the trend of the electron affinity values exhibited by the halogens, Ts was supposed to have a low propensity to gain one extra electron and complete the valence shell. Fully relativistic calculations confirmed the trend of electron affinity to decrease going down the 17-group and provided actually the negative value of $-0.25 \mathrm{eV}$ for the standard reduction potential $\mathrm{Ts} / \mathrm{Ts}^{-}$. Overall, for Ts has been suggested a metalloid character, similar to that of the previous halogen at Pershina (2014).

At $Z=118(\mathrm{Og})$, the filling of the $\mathrm{p}$ shell is completed, and $O g$ has the close-shell configuration $7 \mathrm{~s}^{2} 7 \mathrm{p}^{2}{ }_{1 / 2} 7 \mathrm{p}^{4}{ }_{3 / 2}$. However, $\mathrm{Og}$ can hardly be considered an inert element as the lighter homologs of the 18-group. For this element, a positive electron affinity of $0.056 \mathrm{eV}$ and an ionization potential of $8.914 \mathrm{eV}$ have been theoretically predicted (Pershina et al. 2008). Whereas the ionization potential of $\mathrm{Og}$ is lower than the one expected from the extrapolation of the measured IPs for elements of the 18-group, the calculated polarizability is found significantly larger. The more efficient van der Waals interactions make the $\mathrm{Og}-\mathrm{Og}$ bonds stronger than the Rn-Rn ones (Smits et al. 2020). All these calculated atomic properties indicate that the relativistic effects on the outer $7 p$ orbitals play in Og an increasingly important role, making this element chemically more reactive than its position in the 18-group would imply (Pershina 2019).

Oganesson $(\mathrm{Og})$, the last man-made element accredited up till now, represents an interesting example of how for heavy elements the chemical/physical properties cannot be merely predicted by a linear extrapolation of data measured for the homologous of the group. To get reliable values of the transition energies, i.e., ionization potentials, excitation energies and electron affinities for transactinides, a successful approach was the development of high precision theoretical methods able to take into account simultaneously relativistic and correlation effects. These methods were applied also to a series of heavy elements for which experimental data were available, demonstrating that the computing tools allow to predict the unmeasured atomic properties of the transactinides with an accuracy of few tens of eV (Hofmann et al. 2016). 


\section{Is there any chance for elements beyond $Z=118$ ?}

These reliable theoretical skills have been further exploited to anticipate the electronic configurations of elements still not generated. Based on Dirac-Fock (DF) calculations of atomic ground states, a periodic table up to $Z=172 \mathrm{had}$ been proposed in 1971 (Fricke et al. 1971). Later, developments of DF method (extended average level DF), allowed to better define the configurations of elements in a future enlarged Table (Pyykkö 2011). In these studies, the building up of the electronic structure of the unknown atoms and their insertion in the Periodic Table have been performed by considering the occurrence of a further type of orbitals, the " $g$ " ones. Therefore, the shell-filling order for the elements $119-172$ is:

$8 \mathrm{~s}<5 \mathrm{~g} \leq 8 \mathrm{p}_{1 / 2}<6 \mathrm{f}<7 \mathrm{~d}<9 \mathrm{~s}<9 \mathrm{p}_{1 / 2}<8 \mathrm{p}_{3 / 2}$.

Following these previsions, elements 119 and 120 would open the 8th period, that starts with the filling of the 8s orbitals. The theoretical studies performed on these elements, homologs of the corresponding metals of the 1- and 2-group, evidence that the relativistic contraction of the $8 \mathrm{~s}$ orbitals would strongly affect their chemical/physical properties (Borschevsky et al. 2013a, b).

As examples, differences are foreseen in the intermetallic $\mathrm{M}-\mathrm{M}$ bonding, with values of dissociation energies for elements 119 and 120 contrasting in both cases the trends established for the homologous of the respective 1- and 2 -group (Pershina et al. 2012). For the next element, $Z=121$, the relativistically stabilized $8 \mathrm{p}_{1 / 2}$ sub-shell would lead to a ground-state electronic configuration $8 \mathrm{~s}^{2} 8 \mathrm{p}^{1}{ }_{1 / 2}$, in contrast with the configuration extrapolated from the last two homologous of the 3-group, Ac and La (Eliav et al. 1998a).

The building up of the element 122 would occur by addition of a $7 \mathrm{~d}$ electron, so it is predicted to have an $8 \mathrm{~s}^{2} 7 \mathrm{~d}^{1} 8 \mathrm{p}^{1}$ ground state different from the $7 \mathrm{~s}^{2} 6 \mathrm{~d}^{2}$ state of Th. This is the last element for which accurate Dirac-Coulomb-Breit (DCB) calculations have been performed. For heavier elements, the computation becomes more complicated because of the existence of the $5 \mathrm{~g}$ orbitals and of the fact that all the involved orbitals (7d, 6f, 5g, 9s, 9p, 8p) have very close energies, so that to form a quasi-continuum where the levels are no more clearly distinguishable.

For elements with $Z>122$, a series of calculations and data interpretations suggested over the years various schemes of electronic ground-states arrangements, but the task to place them in an expanded Table is far from being completed. A rather conclusive outcome based on quantum electrodynamics asserted that the Periodic Table should end at $Z=173$ (Greiner et al. 1985). However, this prediction has been further restricted by more recent considerations, pointing that repulsive Coulomb effects will set the limit near $Z=120$ (Möller 2016).

Early predictions of chemical/physical features of SHE played a key role in the planning of the experiments for the synthesis of transactinides, and are thought to be more and more fundamental for the discovery of elements heavier than $Z=118$. This rests, indeed, a very intriguing task in spite of the several demanding attempts ongoing in highlevel nuclear centers. In particular, at GSI, from 2012, Ti beams bombarding ${ }^{249} \mathrm{Bk}$ or ${ }^{249} \mathrm{Cf}$ targets are being used in searching for elements 119 and 120 , respectively, whereas at Dubna and Riken, the discovery of 119 element is currently attemped using a vanadium $(\mathrm{V})$ beam and $\mathrm{Cm}$ targets. So far, all the experiments were not successful.

Both computational studies and experimental evidences demonstrated that the synthesis of the 8s-block elements will be much more challenging than that of the previous artificial elements, and that the current technologies had likely reached their limits. The hope of positive outputs going toward the north edge of the nuclide chart lies in new powerful facilities, like the Superheavy Element Factory opened at Dubna in March 2019, where the exceptional technical parameters of the DC-280 cyclotron are expected to open brand-new opportunities for production of the problematic elements 119 and 120 (Gulbekian et al. 2019).

However, considering that with $Z$ increasing, the production cross sections strongly decrease, even using more intense beams, such nuclei would be generated at a rate still lower than the one of Ts (1 atom/week) or of Og (1 atom/ month). Moreover, the ever-increasing rate of spontaneous fission with $Z$ suggests that the half-lives of any produced 119 or 120 isotope would range from milliseconds down to microseconds, making very hard to experimentally investigate such nuclei and rather impossible those with larger $Z$.

Although the researches for elements 119 and 120 are not being stopped, in some laboratories, a revision of objectives is going on. Instead of continuing with the hunt for still unknown elements, investigations are focused on the already discovered SHEs for which only very limited experimental data are available. And, in particular, efforts are spent trying to produce neutron-rich isotopes of elements with $Z$ in the 106-114 range. These elements are indeed the more close to the regions of the nuclide chart where peculiar ground-state shell effects could make isotopes of superheavy elements unexpectedly stable against spontaneous fission.

\section{Understanding technological limits: a new twist}

A strong experimental evidence in favor of such hypothetical island of stability has been provided by the discovery of elements with $Z>104$, that ideally should not exist at all, and, in 
particular, by the production of some isotopes with lifetimes remarkably longer than the predicted (Möller 2016). The most convincing evidence would be provided by the creation of the double-magic ${ }^{298} \mathrm{Fl}$ isotope with $Z=114$ and $N=184$. But, in the meantime, promising signals come from some isotopes of Tennessine $(Z=117)$. For ${ }^{293}$ Ts $(N=176)$ and ${ }^{294}$ Ts $(N=177)$, half-lives of $25 \mathrm{~ms}$ and $70 \mathrm{~ms}$, respectively, have been measured (Kondev et al. 2021), showing that such isotopes survive much more than expected (Oganessian et al. 2017).

This and other experimental findings seemed to confirm that, for nuclei with $Z$ and $N$ approaching the "magic" numbers, shell effects are able to counteract the instability due to Coulomb repulsion and to reverse the trend exhibited by the $Z>96$ elements of a nuclear stability decreasing with $Z$ increasing. A first glance at Fig. 4 highlights that the existence of hypothetical, long-living, quasi-stable SHEs would be limited to a restricted region of the nuclide chart, localized in the neutron-rich right side of the stability line. Whereas the prediction for stability islands in the nuclide chart opened an exciting scenario on the extreme limit of existence of matter, the moving from conceptualization to experiments was found extremely hard. To get as close as possible to these regions demonstrated to be an insurmountable difficulty using any of the standard types of nuclear reactions used so far to produce artificial elements.

Fission and fragmentation, the only reactions able to generate neutron-rich nuclides, are limited to production of elements up to $Z=92$. On the other hand, the various fusion reactions that had allowed to synthesize superheavy nuclei and discover the transactinides, are intrinsically inadequate to produce isotopes with the neutron richness matching the theoretical requirements. Fusion reactions could produce isotopes with at most 177 neutrons, a result achieved only in ${ }^{294} \mathrm{Ts}$ and ${ }^{293} \mathrm{Lv}$, the isotopes most close to the foreseen stability region among all the discovered ones (Oganessian and

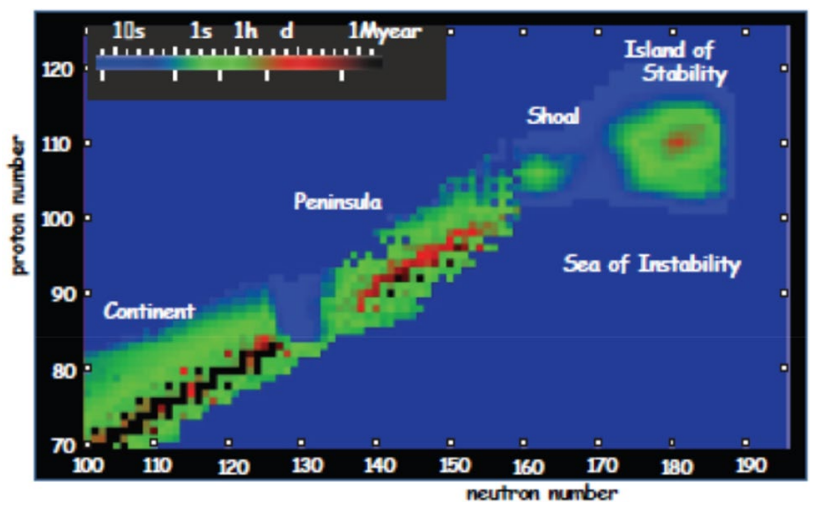

Fig. 4 A picture clearly evidencing the regions of existence for isotopes with $Z>70$ and $N>100$. The blue area indicates the "sea of instability" where elements can not exist. The color scale from blue to black displays the increase in life-time [Courtesy by Yu.Ts. Oganessian, reproduced from Oganessian (2019), pag. 06] (Color figure online)
Utyonkov 2015). Therefore, to go further inside the neutronrich region and to approach as much as possible the stability island, different reaction mechanisms are required and the challenge is the development of novel nuclear paths.

In this context, it has been thought that a chance could be given by the deep inelastic collisions occurring in the so-called multi-nucleon transfer (MNT) reactions, processes applied in the 1960s to produce neutron-rich isotopes of elements up to Th (Fowler et al. 1986). The mechanism of such reactions is based on the formation of "molecular-like" projectile-target systems. This first step is followed by a large exchange of nucleons between the two interacting nuclei (Adamian et al. 2020a). Recent experiments carried out at GSI produced new neutron-deficient isotopes of some transuranium elements, but model calculations predict that MNT reactions could enable to produce also the more long-living SHE isotopes located around the stability island (Adamian et al. 2020b).

\section{Emerging questions, new horizons}

Although in the exhibit room of RIKEN Nishina Center is displayed a LEGO-made three-dimensional nuclide chart showing the many possible combinations of protons and neutrons (En'yo 2019), the creation of new elements is not like a children construction game. As proven by more than seventy years of experiments, immense technical difficulties are encountered trying to merge two nuclei with the proper number on neutrons and protons to produce a nucleus in the upper part of the chart. But the studies had never been stopped. Conversely, computational and experimental researches were strongly pushed on, because there are several significant scientific aspects related to the discovery of elements at the edge of the periodic table.

A fundamental point to be assessed is how much the trends of properties and chemical behavior along groups are still fullfilled in superheavy elements. Up to about element 120 , the deviations produced by relativistic effects influence certainly the chemical properties, but do not seem to completely destroy the systematic ordering of the elements, that still matches their atomic configurations.

For $Z>120$, conversely, the extreme closing of the orbitals energy is expected to interrupt the chemical periodicity (Jerabek et al. 2018).

This expectation arises a basic question related to the existence of a boundary not only for the table but rather for the elements themselves. If in an atom the outermost electrons are no more described by a well defined configuration, and if there are no real shells to speak, the very concept of chemical element vanishes. Moreover, atoms that decay with times too short to enable any chemical process can hardly be qualified as real elements. In this view, IUPAC recalled in May 2018 (Hofmann et al. 2018) that only atoms with 
lifetime longer than $10^{-14} \mathrm{~s}$ may be considered elements. By the way, this threshold is demeed too broad by some chemists. So, one hypothesis is that nuclear experiments designed to produce new nuclei at the farthest extremes of the nuclide chart would lead more to dense aggregates of nucleons than to chemical elements with properties matching their position in an expanded periodic table.

The other hypothesis is that at the end the close cooperation of scientists all around the world will enable to reach the long-sought stability island predicted by theoretical studies, and that new, long-lived superheavy elements will be created. Whether such inspired goal will be reached or not, the search for elements at the limit of the periodic system has in any case achieved, and is still achieving, high returns in both technology and basic sciences.

The large amount of experimental data provided a direct check of the basic statements of nuclear theories (Seyydi 2021), and projected the present nuclear technology toward the future scenario of an even more enlarged table of the elements. Until now, the table demonstrated to be a valid tool in ordering the more than 50 natural or artificial elements discovered during the past one and half centuries (Eichler 2019b; Scerri and Restrepo 2019). Notably, it is impressive that also the various transactinides could be added to the groups of the original Mendeleev's scheme on the basis of their electronic configurations deduced from strenuous, challenging chemical experiments (Ball 2019). But the outputs of the theoretical and experimental studies about superheavy nuclei can open also somewhat unexpected scenarios in astrophysics.
The prevision of nuclear properties for 9318 isotopes of 129 elements with $8 \leq Z \leq 136$ (Möller et al. 2016), and the successful creation of elements at the far end of the Periodic Table by high-energy nuclear reactions, leave open the question if still unknown intriguing nuclei could be present somewhere in the Universe. It is to be noted that some models predict lifetimes up to millions of years for the hypothetical nuclei that mankind is trying to produce right now, but that may have been generated through $\mathrm{rp}, \mathrm{r}$ or spaths, nuclear processes occurring under the strong conditions of the early galactic history. It is to be remembered that the sites for the $r$ processes, that produced the naturally existing heavy nuclei, are still unknown, and that their identification is among the eleven great opened questions of the twenty-first century (National Research Council 2003).

By the discovery of unknown elements, the modern researchers realized the old dream of alchemists that over many centuries attemped to create precious metals by chemical reactions, but the relevant scientific outputs gave a chance to look deeper in our concept of matter at extreme boundaries, and the scientific community has already obtained something much more valuable than gold.

\section{Appendix 1}

\section{See Table 1.}

Table 1 The 15 superheavy elements SHEs (transactinides) known until now

\begin{tabular}{lllllll}
\hline Element & $Z$ & Symbol & $\begin{array}{l}\text { Predicted electron } \\
\text { configuration }\end{array}$ & Mass number $^{\mathrm{b}}$ & Half-life & Decay mode \\
\hline Rutherfordium & 104 & $\mathrm{Rf}$ & $\{\ldots \ldots\} 6 \mathrm{~d}^{2} 7 \mathrm{~s}^{2}$ & 267 & $2.5 \mathrm{~h}$ & $\mathrm{sf}$ \\
Dubnium & 105 & $\mathrm{Db}$ & $\{\ldots \ldots\} 6 \mathrm{~d}^{3} 7 \mathrm{~s}^{2}$ & 268 & $29 \mathrm{~h}$ & $\mathrm{sf}$ \\
Seaborgium & 106 & $\mathrm{Sg}$ & $\{\ldots \ldots\} 6 \mathrm{~d}^{4} 7 \mathrm{~s}^{2}$ & 269 & $5 \mathrm{~min}$ & $\alpha$ \\
Bohrium & 107 & $\mathrm{Bh}$ & $\{\ldots \ldots\} 6 \mathrm{~d}^{5} 7 \mathrm{~s}^{2}$ & 270 & $3.8 \mathrm{~min}$ & $\alpha$ \\
Hassium & 108 & $\mathrm{Hs}$ & $\{\ldots \ldots\} 6 \mathrm{~d}^{6} 7 \mathrm{~s}^{2}$ & $277^{\mathrm{m}}$ & $34 \mathrm{~s}$ & $\mathrm{sf}$ \\
Meitnerium & 109 & $\mathrm{Mt}$ & $\{\ldots \ldots\} 6 \mathrm{~d}^{7} 7 \mathrm{~s}^{2}$ & 278 & $6 \mathrm{~s}$ & $\alpha$ \\
Darmstadtium & 110 & $\mathrm{Ds}$ & $\{\ldots \ldots\} 6 \mathrm{~d}^{9} 7 \mathrm{~s}^{1}$ & 281 & $14 \mathrm{~s}$ & $\alpha 10 \% \mathrm{sf} 90 \%$ \\
Roentgenium & 111 & $\mathrm{Rg}$ & $\{\ldots \ldots\} 6 \mathrm{~d}^{10} 7 \mathrm{~s}^{1}$ & 282 & $2.2 \mathrm{~min}$ & $\alpha$ \\
Copernicium & 112 & $\mathrm{Cn}$ & $\{\ldots \ldots\} 6 \mathrm{~d}^{10} 7 \mathrm{~s}^{2}$ & 285 & $30 \mathrm{~s}$ & $\alpha$ \\
Nihonium & 113 & $\mathrm{Nh}$ & $\{\ldots \ldots\} 6 \mathrm{~d}^{10} 7 \mathrm{~s}^{2} 7 \mathrm{p}^{1}$ & 286 & $12 \mathrm{~s}$ & $\alpha$ \\
Flerovium & 114 & $\mathrm{Fl}$ & $\{\ldots \ldots\} 6 \mathrm{~d}^{10} 7 \mathrm{~s}^{2} 7 \mathrm{p}^{2}$ & 290 & $80 \mathrm{~s}$ & $\alpha$ \\
Muscovium & 115 & $\mathrm{Mc}$ & $\{\ldots \ldots\} 6 \mathrm{~d}^{10} 7 \mathrm{~s}^{2} 7 \mathrm{p}^{3}$ & 290 & $0.84 \mathrm{~s}$ & $\alpha$ \\
Livermorium & 116 & $\mathrm{Lv}$ & $\{\ldots \ldots\} 6 \mathrm{~d}^{10} 7 \mathrm{~s}^{2} 7 \mathrm{p}^{4}$ & 293 & $70 \mathrm{~ms}$ & $\alpha$ \\
Tennessine & 117 & $\mathrm{Ts}$ & $\{\ldots \ldots\} 6 \mathrm{~d}^{10} 7 \mathrm{~s}^{2} 7 \mathrm{p}^{5}$ & 294 & $70 \mathrm{~ms}$ & $\alpha$ \\
Oganesson & 118 & $\mathrm{Og}$ & $\{\ldots \ldots\} 6 \mathrm{~d}^{10} 7 \mathrm{~s}^{2} 7 \mathrm{p}^{6}$ & 295 & $0.68 \mathrm{~s}$ & $\alpha$ \\
\hline
\end{tabular}

${ }^{\mathrm{a}}\{\ldots \ldots\}$ corresponds to the configuration $[\mathrm{Rn}] 5 \mathrm{f}^{14}$ of the core 100 electrons in the SHEs (see Hoffman et al, 2006)

${ }^{\mathrm{b}}$ Refers to the longest half-life identified isotope

${ }^{c}$ Data taken from Kondev et al (2021); decay modes: $\alpha$ (alpha decay), sf (spontaneous fission) 


\section{Declarations}

Conflict of interest The authors did not receive support by any organization for the submitted work, and declare no financial interest or competing interest.

Open Access This article is licensed under a Creative Commons Attribution 4.0 International License, which permits use, sharing, adaptation, distribution and reproduction in any medium or format, as long as you give appropriate credit to the original author(s) and the source, provide a link to the Creative Commons licence, and indicate if changes were made. The images or other third party material in this article are included in the article's Creative Commons licence, unless indicated otherwise in a credit line to the material. If material is not included in the article's Creative Commons licence and your intended use is not permitted by statutory regulation or exceeds the permitted use, you will need to obtain permission directly from the copyright holder. To view a copy of this licence, visit http://creativecommons.org/licenses/by/4.0/.

\section{References}

Adamian GG, Antonenko NV, Diaz-Torres A, Heinz S (2020a) How to extend the chart of nuclides? Eur Phys J A 56:47

Adamian G, Antonenko N, Diaz-Torres A, Heinz S (2020b) How does one extend the chart of nuclides. Nucl Phys News 30:22-26

Aksenov NV, Steinegger P, Abdullin FS et al (2017) On the volatility of nihonium (Nh, $Z=113$ ). Eur. Phys J A Hadrons Nuclei 53:158

Anjali KP, Prathapan K, Biju RK (2020) Studies on the emission of various exotic fragments from superheavy nuclei via cluster decay process. Nucl Phys A 993:121644

Armbruster P, Agarwal YK, Brüchle W et al (1985) Attempts to produce superheavy elements by fusion of ${ }^{48} \mathrm{Ca}$ with ${ }^{248} \mathrm{Cm}$ in the bombarding energy range 4.5-5.2 MeV/u. Phys Rev Lett $54: 406-409$

Ascenzi P, Bettinelli M, Boffi A, Botta M, De Simone G, Luchinat C, Marengo E, Mei H, Aime S (2020) Rare earth elements (REE) in biology and medicine. Rend Fis Acc Lincei 31:821-833

Ball P (2019) Extreme chemistry: experiments at the edge of the periodic table. Nature 565:552-555

Bohr N, Wheeler JA (1939) The mechanism of nuclear fission. Phys Rev 56:426-450

Bolsterli M, Fiset EO, Nix JR, Norton JL (1972) New calculation of fission barriers for heavy and superheavy nuclei. Phys Rev C 5:1050-1077

Borschevsky A, Pershina V, Eliav E, Kaldor U (2009) Electron affinity of element 114 , with comparison to $\mathrm{Sn}$ and $\mathrm{Pb}$. Chem Phys Lett 480:49-51

Borschevsky A, Pershina V, Eliav E, Kaldor U (2013a) Ab initio predictions of atomic properties of element 120 and its lighter group-2 homologues. Phys Rev A 87:022502

Borschevsky A, Pershina V, Eliav E, Kaldor U (2013b) Ab initio studies of atomic properties and experimental behavior of element 119 and its lighter homologs. J Chem Phys 138:124302

Borschevsky A, Pasteka LF, Pershina V, Eliav E, Kaldor U (2015) Ionization potentials and electron affinities of the superheavy elements $115-117$ and their sixth-row homologues Bi, Po, and At. Phys Rev A 91:020501

Brack M, Damgaard J, Jensen AS, Pauli HC, Strutinsk VM, Wong CY (1972) Funny hills: the shell correction approach to nuclear shell effects and its applications to the fission process. Rev Mod Phys 44:320-405

Broglia RA (2002) The surfaces of compact systems: from nuclei to stars. Surf Sci 500:759-792
Cwiok S, Heenen P-H, Nazarewicz W (2005) Shape coexistence and triaxiality in the superheavy nuclei. Nature 433:705-709

Dean DJ (2007) Beyond the nuclear shell model. Phys Today 60:48-53

Deng J-G, Zhang H-F, Royer G (2020) Improved empirical formula for $\alpha$-decay half-lives. Phys Rev C 101:034307

Dobaczewski J, Flocard H, Treiner J (1984) Hartree-Fock-Bogolyubov description of nuclei near the neutron-drip line. Nucl Phys A 422:103-139

Duarte SB, Tavares OAP, Gonçalves M, Rodríguez O, Guzmán F, Barbosa TN, García F, Dimarco A (2004) Half-life predictions for decay modes of superheavy nuclei. J Phys G Nucl Part Phys 30:1487-1494

Dzuba VA (2016) Ionization potentials and polarizabilities of superheavy elements from $\mathrm{Db}$ to $\mathrm{Cn}(\mathrm{Z}=105-112)$. Phys Rev A 93:032519

Eichler R (2019a) The periodic table of elements: Superheavy in chemistry. Nucl Phys News 29:11-15

Eichler R (2019b) The periodic table_-an experimenter's guide to transactinide chemistry. Radiochim Acta 107:865-877

Eliav E, Kaldor U, Ishikawa Y (1995) Transition energies of mercury and ekamercury (element 112) by the relativistic coupled-cluster method. Phys Rev A 52:2765-2769

Eliav E, Shmulyian S, Kaldor U, Ishikawa Y (1998a) Transition energies of lanthanum, actinium, and eka-actinium (element 121). J Chem Phys 109:3954-3958

Eliav E, Kaldor U, Ishikawa Y (1998b) The relativistic coupled-cluster method: transition energies of bismuth and eka-bismuth. Mol Phys 94:181-187

Eliav E, Fritzsche S, Kaldor U (2015) Electronic structure theory of the superheavy elements. Nucl Phys A 944:518-550

En'yo H, (2019) History of nihonium. Pure Appl Chem 91:1949-1958

Faegri K Jr, Trond S (2001) Diatomic molecules between very heavy elements of group 13 and group 17: study of relativistic effects on bonding. J Chem Phys 115:2456-2464

Flerov GN, Oganessian YT, Lobanov YV, Kusnetsov VI, Druin VA, Perelygin VP, Gavrilov KA, Tretiakova SP, Plotko VM (1964) Synthesis and physical identification of the isotope of element 104 with mass number 260. Phys Lett 13:73-75

Forsberg U, Rudolph D, Fahlander C et al (2016) A new assessment of the alleged link between element 115 and element 117 decay chains. Phys Lett B 760:293-296

Fowler MM, Daniels WR, von Gunten HR et al (1986) Radiochemical separation actinides from copper and gold catcher foils from heavy ion reactions with actinide targets. Radiochim Acta 40:75-80

Fricke B, Greiner W, Waber JT (1971) The continuation of the periodic table up to $Z=172$. The chemistry of superheavy elements. Theor Chim Acta 21:235-260

Fricke B, Johnson E, Rivera GM (1993) Ionization potentials and radii of atoms and ions of element 105 (unnilpentium) and ions of tantalum derived from multiconfiguration Dirac-Fock calculations. Radiochim Acta 62:17-26

Gäggler HW, Türler A (2014) Gas-phase, chemistry of superheavy elements. In: Schädel M, Shaughnessy D (eds) The chemistry of the superheavy elements, 2nd edn. Springer, Heidelberg, pp 415-484

Gamow G (1930) Mass defect curve and nuclear constitution. Proc R Soc (lond) A 126:632-644

Ghiorso A, Sikkeland T, Larsh AE, Latimer RM (1961) New element, lawrencium, atomic number 103. Phys Rev Lett 6:473-475

Ghiorso A, Nurmia M, Eskola K, Eskola P (1971) Two new alphaparticle emitting isotopes of element $105,{ }^{261} \mathrm{Ha}$ and ${ }^{262} \mathrm{Ha}$. Phys Rev C 4:1850-1855

Ghiorso A, Nitschke JM, Alonso JR, Alonso CT, Nurmia M, Seaborg GT, Hulet EK, Lougheed RW (1974) Element 106. Phys Rev Lett 33:1490-1493 
Ginges JSM, Dzuba VA (2015) Spectra of barium, radium, and element 120; application of the combined correlation-potential, siglesdoubles, and configuration interaction ab initio methods. Phys Rev A 91:042505

Glebov VA, Kasztura L, Nefedov VS, Zhuikov BL (1989) Is elemento 104 (kurchatovium) a p-element? Radiochim Acta 46:117-121

Greiner W, Müller B, Rafelski J (1985) Quantum electrodynamics of strong fields. Springer, Berlin

Gulbekian GG, Dmitriev SN, Itkis MG, Oganessyan YT et al (2019) Start-up of the DC-280 cyclotron, the basic facility of the factory of superheavy elements of the laboratory of nuclear reactions at the Joint Institute for Nuclear Research. Phys Part Nuclei Lett. $16: 866-887$

Gyanchandani J, Sikka SK (2011) Physical properties of the 6d-series elements from density functional theory: close similarity to lighter transition metals. Phys Rev B 83:172101

Hoffman DC, Lawrence FO, Mewherter JL, Rourke FM (1971) Detection of plutonium-244 in Nature. Nature 234:132-134

Hoffman DC, Lee DM, Pershina V (2006) Transactinides and the future elements. In: Morss E, Norman M, Fuger J (eds) The chemistry of the actinide and transactinide elements, 3rd edn. Springer, Dordrecht

Hofmann S (2010) Welcome copernicium? Nat Chem 2:146

Hofmann S (2016) The discovery of elements 107 to 112. Eur Phys J Web Conf 131:06001

Hofmann S, Ninov V, He $\beta$ berger FP et al (1995a) Production and decay of ${ }^{269} 110$. Z Phys A 350:277-280

Hofmann S, Ninov V, Heßberger FP et al (1995b) The new element 111. Z Phys A 350:281-282

Hofmann S, Ninov V, He $\beta$ berger FP et al (1996) The new element 112. Z Phys A 354:229-230

Hofmann S, Heßberger FP, Ackermann D et al (2002) New results on elements 111 and 112. Eur Phys J A 14:147-157

Hofmann S, Heinz S, Mann R et al (2016) Review of even element super-heavy nuclei and search for element 120 . Eur Phys J A $52: 180$

Hofmann S, Dmitriev SN, Fahlander C, Gates JM, Roberto JB, Sakai H (2018) On the discovery of new elements (provisional report of the 2017 joint working group of IUPAC and IUPAP). Pure Appl Chem 90(11):1773-1832

Itkis MG, Oganessian YT, Zagrebaev V (2002) Fission barriers of superheavy nuclei. Phys Rev C 65:044602

Jerabek P, Schuetrumpf B, Schwerdtfeger P, Nazarewicz W (2018) Electron and nucleon localization functions of oganesson: approaching the Thomas-Fermi limit. Phys Rev Lett 120:053001

Johnson E, Fricke B, Keller OL Jr, Nestor CW, Tucker TC (1990) Ionization potentials and radii of atoms and ions of element 104 (unnilquodium) and hafnium $\left(2^{+}\right)$derived from multiconfiguration Dirac-Fock calculations. J Chem Phys 93:8041-8050

Johnson E, Pershina V, Fricke B (1999) Ionization potentials of seaborgium. J Phys Chem A 103:8458-8462

Johnson E, Fricke B, Jacob T, Dong CZ, Fritzsche S, Pershina V (2002) Ionization potentials and radii of neutral and ionized species of element 107 (bohrium) and 108 (hassium) from extended multiconfiguration Dirac-Fock calculations. J Chem Phys 116:1862-1868

Karol PJ, Barber RC, Sherrill BM, Vardaci E, Yamazaki T (2016) Discovery of the element with atomic number $Z=118$ completing the 7th row of the periodic table (IUPAC technical report). Pure Appl Chem 88:155-160

Khuyagbaatar J, Yakushev A, Düllmann CE et al (2019) Fusion reaction ${ }^{48} \mathrm{Ca}+{ }^{249} \mathrm{Bk}$ leading to formation of the element Ts $(\mathrm{Z}=$ 117). Phys Rev C 99:054306

Kondev FG, Wang M, Huang WJ, Naimi S, Audi G (2021) The NUBASE2020 evaluation of nuclear physics properties. Chin Phys C 45:030001
Kragh H (2018) From transuranic to superheavy elements: a story of dispute and creation, 1st edn. Springer, Berlin

Kratz V, Nagame Y (2014) Liquid-phase chemistry of superheavy elements. In: Schädel M, Shaughnessy D (eds) The chemistry of the superheavy elements, 2nd edn. Springer, Heidelberg, pp 309-371

Magill J, Dreher R, Zs Sòti (2018) Karlsruhe Nuklidkarte, 10th edn. Nucleonica $\mathrm{GmbH}$

McMillan E, Abelson PH (1940) Radioactive element 93. Phys Rev 57:1185-1186

Mendeleev D (1869) The relation of properties with atomic weight of elements. J Russ Chem Soc 1:60-77 (in Russian, on the relations of the properties to the atomic weights of the elements. Z Chim 12: 405 (in German))

Möller P (2016) The limits of the nuclear chart set by fission and alpha decay. Eur Phys J Web Conf 131:03200

Möller P, Sierk AJ, Ichikawa T, Iwamoto A, Bengtsson R, Uhrenholt H, Aberg S (2009) Heavy element fission-barriers. Phys Rev C 79:064304

Möller P, Sierk AJ, Ishikawa T, Sagawa H (2016) Nuclear ground-state masses and deformations: FRDM (2012). At Data Nucl Data Tables 109-110:1-204

Morita K (2015) SHE research at RIKEN/GARIS. Nucl Phys A 944:30-61

Morita K, Morimoto K, Kaji D et al (2007) Experiment on synthesis of an isotope ${ }^{277} 112$ by ${ }^{208} \mathrm{~Pb}+{ }^{70} \mathrm{Zn}$. J Phys Soc Jpn 76:043201

Mosel U, Greiner W (1969) On the stability of superheavy nuclei against fission. Z Phys 222:261-282

Moseley HGJ (1913) The high-frequency spectra of the elements. Philos Mag 26:1024-1032 (ibdem (1914) 27: 703-713)

Münzenberg G (1988) Recent advances in the discovery of transuranium elements. Rep Prog Phys 51:57-104

Münzenberg G, Hofmann S, He $\beta$ berger FP et al (1981) Identification of element 107 by $\alpha$ correlation chains. Z Phys A at Nuclei 300:107-108

Münzenberg G, Armbruster P, Heßberger FP et al (1982) Observation of one correlated $\alpha$-decay in the reaction ${ }^{58} \mathrm{Fe}$ on ${ }^{209} \mathrm{Bi} \rightarrow{ }^{267} 109$. Z Phys A 309:89-90

Münzenberg G, Armbruster P, Folger H et al (1984) The identification of element 108. Z Phys A at Nuclei 317:235-236

Myers WD, Swiatecki WJ (1966) Nuclear masses and deformations. Nucl Phys 81:1-60

Myers WD, Swiatecki WJ (1997) The congruence energy: a contribution to nuclear masses, deformation energies and fission barriers. Nucl Phys A 612:249-261

Nagame Y, Kratz JV, Schädel M (2015) Chemical studies of elements with $Z \geq 104$ in liquid phase. Nucl Phys A 944:614-639

National Research Council (2003) Connecting quarks with the cosmos: eleven science questions for the new century. The National Academies Press, Washington DC

Oganessiam YT, Abdullin FS, Alexander C et al (2013) Experimental studies of the ${ }^{249} \mathrm{Bk}+{ }^{48} \mathrm{Ca}$ reaction including decay properties and excitation function for isotopes of element 117, and discovery of the new isotope ${ }^{277}$ Mt. Phys Rev C 87:054621

Oganessian $\mathrm{Yu}$ (2006) Synthesis and decay properties of superheavy elements. Pure Appl Chem 78:889-904

Oganessian YT (2019) Superheavy elements: on the 150th anniversary of the discovery of the periodic table of elements. Nucl Phys News 29:5-10

Oganessian YT, Rykaczewski KP (2015) A beachhead on the island of stability. Phys Today 68:32-33

Oganessian YT, Utyonkov VK (2015) Super-heavy element research. Rep Prog Phys 78:036301

Oganessian YT, Tret'yakova YP, Il'inov AS et al (1974) Synthesis of neutron-deficient isotopes of fermium, kurchatovium, and element 106. JETP Lett 20:265-266 
Oganessian YT, Demin AG, Danilov NA et al (1976) On spontaneous fission of neutron-deficient isotopes of elements 103, 105 and 107. Nucl Phys A 273:505-522

Oganessian YT, Yeremin AV, Popeko AG et al (1999) Synthesis of nuclei of the superheavy element 114 in reactions induced by ${ }^{48} \mathrm{Ca}$. Nature 400:242-245

Oganessian YT, Utyonkov VK, Lobanov YV et al (2000) Observation of the decay of ${ }^{292} 116$. Phys Rev C 63:011301

Oganessian YT, Utyonkoy VK, Lobanov YV et al (2004) Experiments on the synthesis of element 115 in the reaction $243 \mathrm{Am}(48 \mathrm{Ca}$, xn)291-x115. Phys Rev C 69:021601

Oganessian YT, Abdullin FSh, Bailey PD et al (2010) Synthesis of a new element with atomic number $Z=117$. Phys Rev Lett 104:142502

Oganessian YT, Sobiczewski A, Ter-Akopian GM (2017) Superheavy nuclei: from predictions to discovery. Phys Scr 92:023003

Pearson JM (2015) On the belated discovery of fission. Phys Today 68:40-45

Pershina V (2014) Theoretical chemistry of the heaviest elements. In: Schädel M, Shaughnessy D (eds) The chemistry of superheavy elements, 2nd edn. Springer, Heidelberg, pp 135-239

Pershina V (2015) Electronic structure and properties of superheavy elements. Nucl Phys A 944:578-613

Pershina V (2019) Relativity in the electronic structure of the heaviest elements and its influence on periodicities in properties. Radiochim Acta 107:833-863

Pershina V (2020) Relativistic effects on the electronic structure of the heaviest elements. Is the periodic table endless? C R Chim 23:255-265

Pershina V, Borschevsky A, Eliav E, Kaldor U (2008) Adsorption of inert gases including element 118 on nobel metal and inet surfaces from ab initio Dirac-Coulomb atomic calculations. J Chem Phys 129:144106

Pershina V, Borschevsky A, Anton J (2012) Fully relativistic study of intermetallic dimers of group- 1 elements $\mathrm{K}$ through element 119 and prediction of their adsorption on noble metal surfaces. J Chem Phys 395:87-94

Pitzer KS (1975) Are elements 112, 114, and 118 relatively inert gases? J Chem Phys 63:1032-1033

Poenaru DN, Gherghescu RA, Greiner W (2012) Cluster decay of superheavy nuclei. Phys Rev C 85:034615

Pyykkö P (1988) Relativistic effects in structural chemistry. Chem Rev 88:563-594

Pyykkö P (2011) A suggested periodic table up to $Z \leq 172$, based on Dirac-Fock calculations on atoms and ions. Phys Chem Chem Phys 13:161-168

Pyykkö P, Desclaux J-P (1979) Relativity and the periodic system of elements. Acc Chem Res 12:276-281

Quagliano JV, Vallarino LM (1973) Chemistry, 3rd edn. Prentice-Hall Inc, Hoboken

Raeder S, Ackermann D, Backe H et al (2018) Probing sizes and shapes of nobelium isotopes by laser spectroscopy. Phys Rev Lett 120:232503

Rao MN, Kuroda PK (1966) Decay constant and mass-yield curve for the spontaneous fission of uranium-238. Phys Rev 147:884-886
Santhosh KP, Nithya C (2018) Systematic studies of $\alpha$ and heavycluster emission from superheavy nuclei. Phys Rev C 97:064616

Santiago RT, Haiduke RLA (2020) Determination of molecular properties for muscovium halides $(\mathrm{McF}$ and $\mathrm{McCl})$. Theor Chem Acc 139:60

Scerri E, Restrepo G (eds) (2019) Mendeleev to Oganesson. A multidisciplinary perspective on the periodic table. Oxford University Press, New York

Schwerdtfeger P, Pasteka LF, Punnet A, Bowman PO (2015) Relativistic and quantum electrodynamic effects in superheavy elements. Nucl Phys A 944:551-577

Seaborg GT (1945) The chemical and radioactive properties of the heavy elements. Chem Eng News 23:2190-2193

Seaborg GT (1963) Man-made transuranium elements. Prentice-Hall Inc, Englewood Cliffs

Seyydi SA (2021) Systematic study of alpha-decay of super-heavy isotopes with $Z=120-126$. Mod Phys Lett A 36:2150033

Silva RJ (2006) Fermium, mendelevium, nobelium, and lawrencium. In: Morrs LR, Edelstein NM, Fuger J (eds) The chemistry of the actinide and transactinide elements. Springer Nature Netherlands, Dordrecht, pp 1621-1651

Smits OR, Mewes J-M, Jerabek P, Schwerdtfeger P (2020) Oganesson: a noble gas element that is neither noble nor a gas. Angew Chem Int Ed 59:23636

Sobiczewski A (2011) Theoretical description of superheavy nuclei. Radiochim Acta 99:395-404

Sobiczewski A, Gareev FA, Kalinkin BN (1966) Closed shells for Z > 82 and $N>126$ in a diffuse potential well. Phys Lett 22:500-502

Strutinsk VM (1967) Shell effects in nuclear masses and deformation energies. Nucl Phys A 95:420-442

Strutinsky VM (1968) "Shells" in deformed nuclei. Nucl Phys A $122: 1-33$

Tavares OAP, Medeiros EL (2012) A simple description of cluster radioactivity. Phys Scr 86:015201

Tavares OAP, Medeiros EL, Terranova ML (2005) Alpha decay halflife of bismuth isotopes. J Phys G Nucl Part Phys 31:129-139

Türler A, Gregorich KE (2014) Experimental techniques. In: Schädel M, Shaughnessy D (eds) The chemistry of the superheavy elements, 2nd edn. Springer, Heidelberg, pp 261-330

Türler A, Pershina V (2013) Advances in the production and chemistry of the heaviest elements. Chem Rev 113:1237-1312

Türler A, Eichler R, Yakushev A (2015) Chemical studies of elements with $Z \geq 104$ in gas phase. Nucl Phys A 944:640-689

Viola VE Jr, Seaborg GT (1966) Nuclear systematics of the heavy elements-II. J Inorg Nucl Chem 28:741-761

von Weizsäcker CF (1935) Zur theorie der kernmassen (On the theory of nuclear masses). Z Phys 96:431-458

Xu C, Ren Z, Guo Y (2008) Competition between $\alpha$ decay and spontaneous fission for heavy and superheavy nuclei. Phys Rev C 78:044329

Publisher's Note Springer Nature remains neutral with regard to jurisdictional claims in published maps and institutional affiliations. 